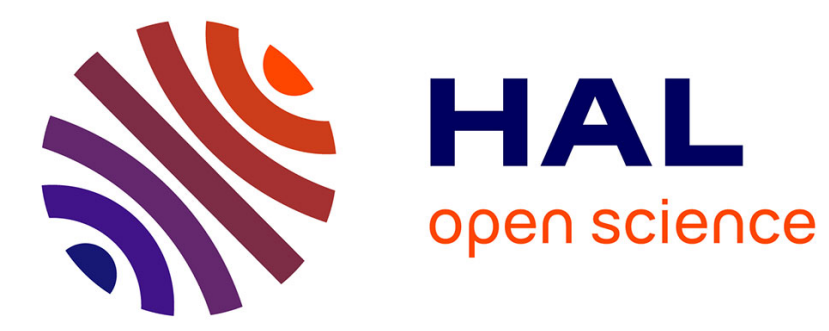

\title{
Asymptotic tracking of a reference trajectory by output-feedback for a class of non linear systems
}

Vincent Andrieu, Laurent Praly, Alessandro Astolfi

\section{To cite this version:}

Vincent Andrieu, Laurent Praly, Alessandro Astolfi. Asymptotic tracking of a reference trajectory by output-feedback for a class of non linear systems. Systems and Control Letters, 2009, 58 (9), pp.652-663. 10.1016/j.sysconle.2009.04.008 . hal-00413103

\section{HAL Id: hal-00413103 https://hal.science/hal-00413103}

Submitted on 3 Sep 2009

HAL is a multi-disciplinary open access archive for the deposit and dissemination of scientific research documents, whether they are published or not. The documents may come from teaching and research institutions in France or abroad, or from public or private research centers.
L'archive ouverte pluridisciplinaire HAL, est destinée au dépôt et à la diffusion de documents scientifiques de niveau recherche, publiés ou non, émanant des établissements d'enseignement et de recherche français ou étrangers, des laboratoires publics ou privés. 


\title{
Asymptotic tracking of a reference trajectory by output-feedback for a class of non linear systems
}

\author{
V. Andrieu* L. Praly ${ }^{\dagger}$ and A. Astolfi ${ }^{\ddagger}$
}

September 3, 2009

\begin{abstract}
We consider the problem of approximately tracking a reference trajectory by means of output feedback for a class of nonlinear systems with some non-globally Lipschitz nonlinearities. We solve this problem combining dynamic scaling, homogeneity in the bi-limit and new small gain arguments.
\end{abstract}

\section{Introduction}

The problem of controlling a system in such a way that its state follows a reference trajectory has been widely studied in control theory and it is still an active field of research (see [7, 8, 13]). When only the output is available for measurement, it can be formulated as follows. Given:

1. a system $\dot{\eta}=f(\eta, u)$ with output $y=h(\eta)$,

2. a bounded state and input reference trajectory $\left(\eta_{r}, u_{r}\right)$, available at each time but not ahead of time

design an output feedback $\dot{w}=\theta\left(w, y, \eta_{r}\right), u=\varphi\left(w, y, \eta_{r}\right)$ which ensures global convergence or, asymptotic closeness (in a sense to be specified) of $\eta$ to $\eta_{r}$.

This problem is challenging since, as shown in [17], controllability and observability are not sufficient to guarantee the existence of a solution, as is the case for linear systems. Hence, some restrictions have to be imposed on the nonlinear function $f$.

Given a state feedback controller, the key step to design an output feedback controller, is the synthesis of an appropriate observer (see [1] for more details). The observer problem is solved in [16, 18, 15], for instance by requiring the nonlinear function $f$ to be linear in the unmeasured variable, or in [13] where $f$ is required to be globally Lipschitz with a Lipschitz constant depending on the output.

\footnotetext{
${ }^{*} \mathrm{~V}$. Andrieu is with LAAS-CNRS, university of Toulouse, France (vincent.andrieu@gmail.com)

${ }^{\dagger}$ L. Praly is with MINES ParisTech, CAS, Mathématiques et Systèmes, 35, rue St-Honoré, 77305 Fontainebleau CEDEX, France (laurent.praly@mines-paristech.fr)

$\ddagger$ A. Astolfi is with the Electrical and Electronic Engineering Dept, Imperial College, London, SW7 2AZ, UK (a.astolfi@ic.ac.uk) and with Dipartimento di Informatica Sistemi e Produzione, University of Rome Tor Vergata, Via del Politecnico 1, 00133 Roma, Italy
} 
Recently, the problem of practical tracking has been solved in 8 for a class of systems not globally Lipschitz in the unmeasured state components, under the assumption the reference trajectory is bounded and this bound is known. The aim of this paper is to extend this result to achieve asymptotic tracking while not requiring the knowledge of an upper-bound for the reference trajectory.

To illustrate the key ideas of the design and to relate our contribution to existing results we first consider an illustrative example円 :

$$
\left\{\begin{array}{l}
\dot{z}=-z+x_{2}^{1+d}, \\
\dot{x}_{1}=x_{2} \\
\dot{x}_{2}=u+x_{2}^{1+d}+z \\
y=x_{1}
\end{array}\right.
$$

where $y$ in $\mathbb{R}$ is the available measurement, $u$ in $\mathbb{R}$ is the control input and $d$ is a real number in $[0,1)$. Given a bounded time function $t \mapsto\left(x_{r 1}(t), x_{r 2}(t), u_{r}(t)\right)$, together with a solution of

$$
\dot{z}_{r}=-z_{r}+x_{2, r}^{1+d}
$$

we define a state and input reference trajectory $\left(\left(z_{r}, x_{r, 1}, x_{r, 2}\right), u_{r}\right)$, which is an approximate solution of (11), i.e. it solves

$$
\left\{\begin{array}{l}
\dot{z}_{r}=-z_{r}+x_{2, r}^{1+d} \\
\dot{x}_{r, 1}=x_{r, 2}+\delta_{r, 1} \\
\dot{x}_{r, 2}=u_{r}+x_{r, 2}^{1+d}+z_{r}+\delta_{r, 2},
\end{array}\right.
$$

where $\left(\delta_{r, 1}, \delta_{r, 2}\right)$ quantifies the approximation error. The problem is to find an output feedback such that the state $\left(z, x_{1}, x_{2}\right)$ of (1) approaches (in a sense to be specified) $\left(z_{r}, x_{r, 1}, x_{r, 2}\right)$ despite the presence of $\left(\delta_{r, 1}, \delta_{r, 2}\right)$.

Note that system (11) is neither linear nor globally Lipschitz with respect to its unmeasured state components due to the presence of the term $x_{2}^{1+d}$. Hence, none of the tracking results developed in 16, 18, 15, 13 can be used. Moreover functions $z_{r}, x_{r, 1}, x_{r, 2}, u_{r}, \delta_{r, 1}$ and $\delta_{r, 2}$ are not required to be bounded in norm by some known quantities, and this impedes the use of the technique proposed in 8$]$.

In this paper, the tracking problem is recast into the problem of finding $\tilde{u}=u-u_{r}$, depending on $\left(z_{r}, x_{r, 1}, x_{r, 2}\right)$ and $\tilde{x}_{1}=x_{1}-x_{r, 1}$ such that the solutions of the error systems

$$
\left\{\begin{array}{l}
\dot{\tilde{z}}=-\tilde{z}+\left(x_{r, 2}+\tilde{x}_{2}\right)^{1+d}-x_{r, 2}^{1+d}, \\
\dot{\tilde{x}}_{1}=\tilde{x}_{2}-\delta_{r, 1} \\
\dot{\tilde{x}}_{2}=\tilde{u}+\left(x_{r, 2}+\tilde{x}_{2}\right)^{1+d}-x_{r, 2}^{1+d}+\tilde{z}-\delta_{r, 2}
\end{array}\right.
$$

asymptotically converges to a ball centered at the origin, with radius depending only on the asymptotic behavior of $\delta_{r, 1}$ and $\delta_{r, 2}$.

To solve this problem we follow a domination approach based on homogeneity. This leads to regard the term $\left(x_{r, 2}+\tilde{x}_{2}\right)^{1+d}-x_{r, 2}^{1+d}+\tilde{z}-\delta_{r, 2}$ in the definition of $\dot{\tilde{x}}_{2}$ as a perturbation which can be upper bounded as :

$$
\left|\left(x_{r, 2}+\tilde{x}_{2}\right)^{1+d}-x_{r, 2}^{1+d}+\tilde{z}-\delta_{r, 2}\right| \leq(1+d)\left|x_{r, 2}\right|^{d}\left|\tilde{x}_{2}\right|+\left|\tilde{x}_{2}\right|^{1+d}+|\tilde{z}|+\left|\delta_{r, 2}\right| .
$$

This bound is composed of four terms each of which motivate some particular features of the proposed design.

\footnotetext{
${ }^{1}$ For any real numbers $w \neq 0$ and $r, w^{r}$ denotes $\operatorname{sign}(w)|w|^{r}$.
} 
1. The term $(1+d)\left|x_{r, 2}\right|^{d}\left|\tilde{x}_{2}\right|$ is a known time function which multiplies a linear function of the tracking error. To deal with this kind of term we follow an idea introduced in 19] and design a high-gain output feedback with a dynamic scaling with a gain updated from the reference signal $x_{r, 2}$.

2. The second term, namely $\left|\tilde{x}_{2}\right|^{1+d}$, is a power of the norm of the tracking error $\left|\tilde{x}_{2}\right|$. To deal with this term, we use the homogeneous in the bi-limit output feedback design tool we have introduced in [2] (see Appendix A for the definition of homogeneity in the bi-limit).

3. The term $|\tilde{z}|$ depends on the state of the appended dynamics. We deal with this one by imposing a minimum phase assumption and invoking a small gain argument. Note that the gain obtained is time-varying (it depends on $x_{r, 2}$ ), and this requires, in the design, to rely on a time-varying small-gain argument.

4. Finally, the term $\left|\delta_{r, 2}\right|$ coming from the approximation error of the reference is a perturbation which is not necessary vanishing at the origin. This implies that exact tracking cannot be obtained. Nevertheless, the use of high gain allows to reduce the effect of this disturbance.

In conclusion, the solution to this tracking problem is based on a domination approach and combines high-gain with dynamic scaling and homogeneity in the bi-limit.

In Section 2 the main result of the paper is stated, commented and compared with existing results related to this topic. Section 3 is devoted to the proof of the main result. More precisely, in Section 3.1 we introduce a homogeneous in the bi-limit output feedback design for a chain of integrators compatible with the use of dynamic scaling. With this tool in hand, we propose an output feedback and adjust some of its parameters by studying the closed-loop system in Section 3.2. A brief summary of the homogeneity in the bi-limit theory is given in Appendix A, while some technical results are proved in Appendix B.

\section{Main result of the paper}

\subsection{Problem statement and Assumptions}

Consider a system whose dynamics are described by :

$$
\left\{\begin{aligned}
\dot{z} & =F(z, x) \\
\dot{x}_{1} & =x_{2}+f_{1}(z, x), \\
\dot{x}_{2} & =x_{3}+f_{2}(z, x), \quad y=x_{1}, \\
& \vdots \\
\dot{x}_{n} & =u+f_{n}(z, x),
\end{aligned}\right.
$$

where $x=\left(x_{1}, \ldots x_{n}\right)$ is in $\mathbb{R}^{n}, y$ is the output in $\mathbb{R}, u$ is the input in $\mathbb{R}$ and $z$ in $\mathbb{R}^{n_{z}}$ is the state of some appended dynamicst.

\footnotetext{
${ }^{2}$ Depending on the structure of the functions $F$ and $f_{i}$ 's these dynamics are usually referred as "inverse dynamics". For instance, this is the case if $F(z, x)=F\left(z, x_{1}\right)$.
} 
Let $t \mapsto\left(x_{r}(t), u_{r}(t)\right) \in \mathbb{R}^{n} \times \mathbb{R}$ be a bounded function to which corresponds a bounded solution $t \mapsto z_{r}(t) \in \mathbb{R}^{n_{z}}$ of the appended state dynamics

$$
\dot{z}_{r}=F\left(z_{r}, x_{r}\right)
$$

We consider $t \mapsto\left(x_{r}(t), z_{r}(t), u_{r}(t)\right) \in \mathbb{R}^{n} \times \mathbb{R}$ as a state and input reference trajectory. It is a solution of (4) up to an approximation error $t \mapsto \delta_{r}(t)=\left(\delta_{r, 1}(t), \ldots, \delta_{r, n}(t)\right) \in \mathbb{R}^{n}$, defined as :

$$
\left\{\begin{aligned}
\delta_{r, 1} & =x_{r, 2}+f_{1}\left(z_{r}, x_{r}\right)-\dot{x}_{r, 1}, \\
\delta_{r, 2} & =x_{r, 3}+f_{2}\left(z_{r}, x_{r}\right)-\dot{x}_{r, 2}, \\
& \vdots \\
\delta_{r, n-1} & =x_{r, n}+f_{n-1}\left(z_{r}, x_{r}\right)-\dot{x}_{r, n-1} \\
\delta_{r, n} & =u_{r}+f_{n}\left(z_{r}, x_{r}\right)-\dot{x}_{r, n}
\end{aligned}\right.
$$

we wish to design an output feedback controller for system (雨) to ensure convergence (or closeness, see inequality (15)) of the solutions $(x, z)$ toward this state reference trajectory $\left(x_{r}, z_{r}\right)$.

In the design, the $z$ part can be "neglected" provided the appended dynamics with $f_{i}$ as output and $x$ as input are incremental ISS (see [6]). Specifically, we make the following assumptionst.

Assumption 1 (Minimum-Phase) There exist non-negative $C^{1}$ functions $Z_{i}$ and $C^{0}$ functions $\gamma_{i}$, such that,

1.1) for each $c \geq 0$, the set $\left\{\tilde{z}: \exists z_{r}:\left|z_{r}\right|+\sum_{i=1}^{n} Z_{i}\left(z_{r}, \tilde{z}\right) \leq c\right\}$ is compact.

1.2) $\frac{\partial Z_{i}}{\partial z_{r}}\left(z_{r}, \tilde{z}\right) F\left(z_{r}, x_{r}\right)+\frac{\partial Z_{i}}{\partial \tilde{z}}\left(z_{r}, \tilde{z}\right)\left[F\left(z_{r}+\tilde{z}, x_{r}+\tilde{x}\right)-F\left(z_{r}, x_{r}\right)\right] \leq$

$$
-Z_{i}\left(z_{r}, \tilde{z}\right)+\gamma_{i}\left(z_{r}, x_{r}, \tilde{x}\right)
$$

As shown in [17] in the context of global asymptotic stabilization of the origin, the tracking problem under consideration might be unsolvable. Consequently, we need to impose some restrictions on the functions $f_{i}$. In this regard, we make the following assumption.

Assumption 2 (Nonlinear bound) : There exist positive real numbers $q_{1}$ and $q_{2}$, a real number $\mathfrak{d}_{\infty}$ in $\left[0, \frac{1}{n-1}\right)$, a positive real number $c_{\infty}$, a non-negative continuous function $\Omega$, and nonnegative functions $\mu_{i}$ such that :

2.1) the functions $\mu_{i}$ are zero at zero, $C^{1}$ on $(0+\infty)$ and continuous at 0 , the functions $\mu_{i}^{q_{1}}$ are convex and :

$$
s \mu_{i}^{\prime}(s) \leq q_{2} \mu_{i}(s) \quad \forall s>0
$$

2.2) for all $i$ in $\{1, \ldots, n\}$ and all $\left(z_{r}, \tilde{z}, x_{r}, \tilde{x}\right)$ in $\mathbb{R}^{2 n_{z}+2 n}$,

$$
\left|f_{i}\left(z_{r}+\tilde{z}, x_{r}+\tilde{x}\right)-f_{i}\left(z_{r}, x_{r}\right)\right| \leq \Omega\left(z_{r}, x_{r}\right) \sum_{j=1}^{i}\left|\tilde{x}_{j}\right|+c_{\infty} \sum_{j=1}^{i}\left|\tilde{x}_{j}\right|^{\frac{1-\mathfrak{d} \infty(n-i-1)}{1-\mathfrak{d} \infty(n-j)}}+\mu_{i}\left(Z_{i}\left(z_{r}, \tilde{z}\right)\right) .
$$

\footnotetext{
${ }^{3}$ With Assumption 1, boundedness of $z_{r}$ is implied by the boundedness of $x_{r}$.

${ }^{4}$ See Section 2.3.2 for some discussions on Assumptions 1, 2 and 3.
} 
Finally, for the appended dynamics, we impose a bound on the gain between its input $x$ and its outputs $f_{i}$.

Assumption 3 (Bound on the gain) There exists a strictly positive real number $v$ such that, for all $i$ in $\{1, \ldots, n\}$ and all $\left(z_{r}, x_{r}, \tilde{x}\right)$ in $\mathbb{R}^{n_{z}+2 n}$, we have,

$$
\mu_{i}\left((1+v) \gamma_{i}\left(\tilde{x}, z_{r}, x_{r}\right)\right) \leq \Omega\left(z_{r}, x_{r}\right) \sum_{j=1}^{i}\left|\tilde{x}_{j}\right|+c_{\infty} \sum_{j=1}^{i}\left|\tilde{x}_{j}\right|^{\frac{1-\mathfrak{d} \infty(n-i-1)}{1-\mathrm{d} \infty(n-j)}}
$$

where the functions $\gamma_{i}, \mu_{i}, \Omega$ and the positive real number $c_{\infty}$ are as in Assumptions 1 and 2.

\subsection{Main result}

For systems satisfying Assumptions 1, 2 and 3, the high-gain output feedback we propose to solve the tracking problem is expressed as :

$$
u=u_{r}+L^{n+b} \phi\left(\mathfrak{L}^{-1} \hat{x}\right), \quad \dot{\hat{x}}=\mathcal{S} \hat{x}+B L^{n+b} \phi\left(\mathfrak{L}^{-1} \hat{x}\right)+L \mathfrak{L} K\left(\frac{\hat{x}_{1}-\left(y-x_{r, 1}\right)}{L^{b}}\right),
$$

where

$$
\mathfrak{L}=\operatorname{diag}\left(L^{b}, \ldots, L^{n+b-1}\right)
$$

$\mathcal{S}$ denotes the left shift matrix of order $n$, i.e.

$$
\mathcal{S} \hat{x}=\left(\hat{x}_{2}, \ldots, \hat{x}_{n-1}, 0\right)^{T}
$$

and $b$ is a positive real number chosen to satisfy Đ, for $1 \leq j \leq i \leq n$,

$$
\frac{1-\mathfrak{d}_{\infty}(n-i-1)}{1-\mathfrak{d}_{\infty}(n-j)}<\frac{i+b}{j-1+b}<\frac{i}{j-1},
$$

for all $1 \leq j \leq i \leq n$ and with $\mathfrak{d}_{\infty}$ as given in Assumption 2. Similarly to [2], the functions $K$ and $\phi$ are designed by following the procedure described in Section 3.1.3. Unlike [2], the high-gain parameter $L$ is updated on line as :

$$
\dot{L}=-a_{1} L+L \max \left\{0, a_{1}\left(a_{2}+1-L^{\epsilon}\right)+a_{3} \bar{\Omega}\left(z_{r}, x_{r}\right)\right\}
$$

where :

$$
\bar{\Omega}\left(z_{r}, x_{r}\right)=\max \left\{\frac{\Omega\left(z_{r}, x_{r}\right)}{c_{\infty}}, 1\right\}^{\frac{d_{U}+\mathrm{d} \infty}{1-\mathfrak{d} \infty(n-2)}},
$$

and where $d_{U}, a_{1}, a_{2}$, and $a_{3}$ are positive real numbers to be defined, with $a_{1}$ sufficiently small and $d_{U}, a_{2}$ and $a_{3}$ sufficiently large, and $\epsilon$ is selected to satisfy, for $1 \leq j \leq i \leq n$,

$$
0<\epsilon<i+b-(b+j-1) \frac{1-\mathfrak{d}_{\infty}(n-i-1)}{1-\mathfrak{d}_{\infty}(n-j)}
$$

\footnotetext{
${ }^{5}$ This choice is always possible since, for $1 \leq j \leq i \leq n$, we have :

$$
\begin{gathered}
\frac{i+b}{j-1+b}<\frac{i}{j-1} \quad \forall b>0 \\
1 \leq \frac{1-\mathfrak{d}_{\infty}(n-i-1)}{1-\mathfrak{d}_{\infty}(n-j)}<\frac{i}{j-1} \quad \forall \mathfrak{d}_{\infty} \in\left[0, \frac{1}{n-1}\right) .
\end{gathered}
$$

and
} 
The update law (11) is a modification of the one introduced in [19, (24)] and in [7, (3.12)] or [14, (134)]. Its right hand side depends only on the state reference trajectory $\left(x_{r}, z_{r}\right)$. Since the reference trajectory is bounded by assumption, $L$ is upper bounded along any closed-loop solution. Moreover, if $L(0)>a_{2}^{\frac{1}{\epsilon}}, L(t)$ remains larger than $a_{2}^{\frac{1}{\epsilon}}$ along any closed-loop solution. In particular, $L(t)>1$ if we select $a_{2} \geq 1$. Finally the presence of the term $-a_{1} L$ allows to recover the main property of [19, (24)], i.e. $L$ "follows" its driving term. Specifically, as established in Appendix B, we have :

$$
\begin{aligned}
& \limsup _{t \rightarrow+\infty} L(t) \leq\left[a_{2}+\frac{a_{3}}{a_{1}} \limsup _{t \rightarrow+\infty}\left\{\bar{\Omega}\left(z_{r}(t), x_{r}(t)\right)\right\}\right]^{\frac{1}{\epsilon}}, \\
& \liminf _{t \rightarrow+\infty} L(t) \geq\left[a_{2}+\frac{a_{3}}{a_{1}} \liminf _{t \rightarrow+\infty}\left\{\bar{\Omega}\left(z_{r}(t), x_{r}(t)\right)\right\}\right]^{\frac{1}{\epsilon}} .
\end{aligned}
$$

We are now ready to state the main result of the paper (proved in Section 3).

Theorem 1 Under Assumptions 1,2 and 3, given any strictly positive real numbers $b$ satisfying (10) and a sufficiently large real number $d_{U}$, there exist a positive real number $c_{r}$ and functions $K$ and $\phi$ such that, for all sufficiently small strictly positive real number $a_{1}$ and sufficiently large real numbers $a_{2}$ and $a_{3}$, the following holds.

For any bounded state and input reference trajectory $t \mapsto\left(x_{r}(t), z_{r}(t), u_{r}(t)\right)$, with bounded approximation error $t \mapsto \delta_{r}(t)$ given by (ब), the solutions of system (四) with the output feedback (8), (11) are bounded in positive time and satisfy

$$
\begin{aligned}
& \limsup _{t \rightarrow+\infty}\left|\mathfrak{L}^{-1}\left(x(t)-x_{r}(t)\right)\right| \leq \\
& c_{r} \limsup _{t \rightarrow+\infty}\left\{\sum_{i=1}^{n-1} \frac{\left|\delta_{r, i}(t)\right|}{L(t)^{i+b}}+\left(\frac{\left|\delta_{r, i}(t)\right|}{L(t)^{i+b}}\right)^{\frac{1}{1-\mathfrak{d} \infty(n-i-1)}}+2 \frac{\left|\delta_{r, n}(t)\right|}{L(t)^{n+b}}\right\}
\end{aligned}
$$

with $\mathfrak{L}$ defined in (9).

\subsection{Some remarks}

\subsubsection{About the result}

If the reference trajectory is an exact solution of (雨) (i.e. if $\delta_{r}(t)=0$ ) or if $\delta_{r}(t)$ converges to zero as $t$ goes to infinity, the output feedback given in Theorem 1 ensures that $(x(t), z(t))$ converges to $\left(x_{r}(t), z_{r}(t)\right)$.

In general, (15) implies

$$
\begin{aligned}
& \limsup _{t \rightarrow+\infty}\left|y(t)-x_{r, 1}(t)\right| \leq \\
& c_{r} \frac{\limsup _{t \rightarrow+\infty} L(t)^{b}}{\liminf _{t \rightarrow+\infty} L(t)^{\frac{1+b}{1+\boldsymbol{d} \infty}}} \limsup _{t \rightarrow+\infty}\left\{\sum_{i=1}^{n-1}\left|\delta_{r, i}(t)\right|+\left|\delta_{r, i}(t)\right|^{\frac{1}{r_{\infty, i}+\mathrm{d} \infty}}+2\left|\delta_{r, n}(t)\right|\right\} .
\end{aligned}
$$

Therefore, if the reference trajectory is known in advance, for all $\kappa>0$ we can select the parameter $a_{2}$ strictly larger than

$$
\limsup _{t \rightarrow+\infty}\left\{\max \left\{\frac{a_{3}}{a_{1}} \bar{\Omega}\left(z_{r}(t), x_{r}(t)\right), c_{r}^{\epsilon}\left(\frac{\sum_{i=1}^{n-1}\left|\delta_{r, i}(t)\right|+\left|\delta_{r, i}(t)\right|^{\frac{1}{r_{\infty, i}+\mathrm{D} \infty}}+2\left|\delta_{r, n}(t)\right|}{\kappa}\right)^{\epsilon} 2^{b}\right\}\right\} .
$$


Then, by (13) and (14),

$$
\limsup _{t \rightarrow+\infty}\left|y(t)-x_{r, 1}(t)\right| \leq \kappa .
$$

Hence, as in [8], we recover a practical result in its usual formulation.

On the other hand, we do not need to know in advance the whole reference trajectory or even a bound on it to design the controller and to get asymptotically closeness of the closed loop system solutions toward the reference one. This differs from the results proposed in [8] and [15].

\subsubsection{About the Assumptions}

Note that for system (四),

- Assumption 1 follows from inequality (3) taking

$$
Z_{1}\left(z_{r}, \tilde{z}\right)=Z_{2}\left(z_{r}, \tilde{z}\right)=|\tilde{z}|^{2}
$$

and

$$
\gamma_{1}\left(\tilde{x}, z_{r}, x_{r}\right)=\gamma_{2}\left(\tilde{x}, z_{r}, x_{r}\right)=\left.|(1+d)| x_{r, 2}\right|^{d}\left|\tilde{x}_{2}\right|+\left.\left|\tilde{x}_{2}\right|^{1+d}\right|^{2}
$$

- Assumption 2 is satisfied setting $\mathfrak{d}_{\infty}=d, c_{\infty}=\sqrt{2}$ and picking

$$
\Omega\left(z_{r}, x_{r}\right)=\sqrt{2}(1+d)\left|x_{r, 2}\right|^{d}, \quad \mu_{1}(s)=\mu_{2}(s)=\sqrt{s}, \quad q_{1}=2, \quad q_{2}=\frac{1}{2} .
$$

- Finally, Assumption 3 is satisfied with $v=1$.

Consequently, system (11) belongs to the class of systems satisfying Assumption 1,2 and 3 and Theorem 1 applies.

When compared with what can be found in the textbooks [18, 16] or in [15 for instance, our approach allows us to deal with dynamics which, in appropriate coordinates, may have some polynomial growth in the unmeasured state components (as expressed by Assumption 2).

Assumption 1 is more general than the incremental property introduced in [6] (see also [3]) since the gain $\gamma_{i}$ in Assumption 1.2 depends also on $\left(z_{r}, x_{r}\right)$. Nevertheless it retains its main property on the behavior of the solutions. Specifically it implies that, for all bounded time functions $t \mapsto\left(\mathfrak{x}(t), \mathfrak{x}_{r}(t)\right)$, if a corresponding solution $t \mapsto \mathfrak{z}_{r}(t)$ of the appended dynamics of system (4), i.e. solution of :

$$
\dot{\mathfrak{z}}_{r}=F\left(\mathfrak{z}_{r}, \mathfrak{x}_{r}\right),
$$

is bounded, then all solutions $t \mapsto \mathfrak{z}(t)$ of :

$$
\dot{\mathfrak{z}}=F(\mathfrak{z}, \mathfrak{x})
$$

are bounded as well. If furthermore, for all $i, \gamma_{i}\left(\mathfrak{z}_{r}(t), \mathfrak{x}_{r}(t), \mathfrak{x}(t)-\mathfrak{x}_{r}(t)\right)$ goes to zero when $t$ goes to infinity, then $\left|\mathfrak{z}(t)-\mathfrak{z}_{r}(t)\right|$ goes to zero.

The presence of the $\delta_{r, i}$ offers a great flexibility for checking our Assumptions. Specifically, it follows from our proof that Assumptions 1 and 2 need not to hold for all $\left(z_{r}, x_{r}\right)$. It is

\footnotetext{
${ }^{6}$ See Section 2.3.2 for more details.
} 
sufficient they are satisfied with $F\left(z_{r}, x_{r}\right)$ and $\delta_{r, i}$ given by (6). For example, in the case with no appended dynamics, by letting :

$$
\delta_{r, 1}=\dot{x}_{r, 1}-f_{1}\left(x_{r}\right) \quad, \quad x_{r, i}=0, \quad \delta_{r, i}=f_{i}\left(x_{r}\right) \quad \forall i \geq 2,
$$

Assumption 2 becomes :

$$
\left|f_{i}(\tilde{x})\right| \leq \Omega\left(x_{r}\right) \sum_{j=1}^{i}\left|\tilde{x}_{j}\right|+c_{\infty} \sum_{j=1}^{i}\left|\tilde{x}_{j}\right|^{\frac{1-\mathfrak{d} \infty(n-i-1)}{1-\mathfrak{d}_{\infty}(n-j)}} .
$$

Then, with $f_{i}$ locally Lipschitz, around the origin, we recover the result on practical tracking in [8] where standard homogeneity and domination is also used.

In the same way, by letting

$$
F\left(z_{r}, x_{r}\right)=0 \quad, \quad \delta_{r, i}=f_{i}\left(0, x_{r}\right)-f_{i}\left(z_{r}, x_{r}\right)
$$

Assumption 1.2 reduces to a simple ISS property as assumed for example in [15].

Finally, the presence of the function $\Omega$ in Assumptions 2 and 3 is an important feature. It is necessary to account for the fact that the local Lipschitz constant depend on around where the solution is and therefore around where $\left(z_{r}, x_{r}\right)$ is. But, not knowing in advance the reference trajectory, we have no a priori upperbound for this term. This term makes the analysis much more involved and requires ad hoc small gain arguments.

\subsubsection{About the reference trajectory}

The standard output tracking problem consists in designing a feedback which guarantees boundedness of the closed loop solutions while ensuring convergence of the output to a desired reference signal $y_{r}$. To solve this problem, we follow the classical two degrees of freedom design technique. Namely we separate the problem into two subproblems :

- trajectory generation,

- feedback compensation.

Feedback compensation is the object we present in full details in this paper. The purpose of trajectory generation is to synthesize a state and input reference trajectory $\left(x_{r}, z_{r}, u_{r}\right)$ for the system given the desired reference signal $y_{r}$. Here we do not require this trajectory to be feasible since a "dynamical mismatch" quantified by $\delta_{r}$ is allowed. In addition we do not require this state and input reference trajectory, and therefore the reference signal to be available ahead of time.

The problem of state and input reference trajectory generation is rendered more difficult by the presence of appended dynamics. When the system we consider is right invertible (see [10]), one way to solve this problem (among many others) is to decompose it in two sub-problems:

1. generation of the time derivatives of the desired reference signal $y_{r}$,

2. transformation of these derivatives into a state and input reference trajectory. 
The problem of obtaining the time derivative of the desired reference signal is an observer problem. It can be solved once we have a model of the system which generates the reference signal, the so-called exo-system in regulation theory [11]. For example, we could pick as approximate exo-system $y_{r}^{(m)}(t)=0$, i.e. $y_{r}(t)$ is approximated as a polynomial of degree $m-1$ in $t$ (the model used in $\mathbb{8}$ is $\dot{y}_{r}=0$ ).

Then, to obtain the appropriate signals $\left(x_{r}, u_{r}\right)$ and $z_{r}$, from $y_{r}$ and its time derivatives $y_{r}^{(i)}$, we follow a standard inversion procedure (see [10]). Specifically, if we can partition $x$ in $\left(x_{a}, x_{b}\right)$ so that (1) takes the specific form?

$$
\left\{\begin{aligned}
\dot{z} & =F\left(z, x_{a}\right) \\
\dot{x}_{a 1} & =x_{a 2}+f_{1}\left(x_{a 1}\right) \\
& \vdots \\
\dot{x}_{a n_{a}-1} & =x_{a n_{a}}+f_{n_{a}-1}\left(x_{a 1}, \ldots, x_{a n_{a}-1}\right), \\
\dot{x}_{a n_{a}} & =x_{b 1}+f_{n_{a}}\left(z, x_{a}\right) \\
\dot{x}_{b 1} & =x_{b 2}+f_{n_{a}+1}\left(z, x_{a}, x_{b 1}\right) \\
& \vdots \\
\dot{x}_{b n_{b}} & =u+f_{n_{a}+n_{b}}\left(z, x_{a}, x_{b 1}, \ldots, x_{b n_{b}}\right)
\end{aligned}\right.
$$

then by formal differentiation we obtain functions $\phi_{a i}$ so that $x_{r, a i}=\phi_{a i}\left(y_{r}, \ldots, y_{r}^{(i-1)}\right)$, and by on-line integration of

$$
\dot{z}_{r}=F\left(z_{r}, x_{r, a}\right)
$$

we obtain $z_{r}$ from $x_{r, a}$. Finally, again by formal differentiation, we obtain functions $\phi_{b i}$ so that

$$
x_{r, b i}=\phi_{b i}\left(z_{r}, y_{r} \ldots, y_{r}^{\left(n_{a}+i-1\right)}\right) \quad, \quad u_{r}=\phi_{b n}\left(z_{r}, y_{r} \ldots, y_{r}^{\left(n_{a}+n_{b}\right)}\right) .
$$

If, for the $z$ subsystem there exist a particular initial condition $z(0)$ and a bounded time function $t \mapsto x_{a}(t)$ (considered here as input) such that the corresponding solution $t \mapsto z(t)$ is bounded, then, by the minimum phase Assumption 1.1.2, the above procedure yields a bounded state and input reference trajectory provided that the function $t \mapsto\left(y_{r}(t), \ldots, y_{r}^{\left(n_{a}+n_{b}\right)}(t)\right)$ is bounded. Note that we rely on a minimum phase assumption and this is not surprising in view of the key role this assumption plays, as discussed in [9].

For system (11) it is possible to compute an exact state and input trajectory from $y_{r}, \dot{y}_{r}$ and $\ddot{y}_{r}$ selecting $x_{a}=\left(x_{1}, x_{2}\right)$ with $x_{b}=\emptyset$. Indeed, we obtain :

$$
x_{r, 2}=\dot{y}_{r} \quad, \quad u_{r}=\ddot{y}_{r}-\left(\dot{y}_{r}\right)^{1+d}-z_{r} \quad, \quad \dot{z}_{r}=-z_{r}+\left(\dot{y}_{r}\right)^{1+d} .
$$

Unfortunately, in general, the estimation process yielding the time function $\dot{y}_{r}(t)$ and $\ddot{y}_{r}(t)$ from the knowledge of $y_{r}(t)$ is not exact, and consequently generates an approximation for this reference trajectory (i.e. non-zero $\delta_{1}$ and $\delta_{2}$ ).

The procedure above is only one of many possible solutions. Others may exploit optimal control or flatness [24.

\section{Proof of Theorem $₫$}

The proof of inequality (15) is divided into two parts. In the first part we introduce the functions $K$ and $\phi$ appearing in the output feedback given in (8). In the second part we show

\footnotetext{
${ }^{7} x_{b}$ is not an argument of $F$ and $z$ is not an argument of $f_{1}, \ldots, f_{n_{a}-1}$.
} 
how a proper selection of the parameters $d_{U}, a_{1}, a_{2}$ and $a_{3}$ in the high-gain update law (11) yields the result.

\subsection{Construction of $K$ and $\phi$}

\subsubsection{Posing the problem as an output feedback stabilization problem}

The first step is to pose the tracking problem as a problem of stabilization by output feedback. Setting

$$
\tilde{u}=u-u_{r}, \quad \tilde{x}=x-x_{r}, \quad \tilde{z}=z-z_{r},
$$

we obtain :

$$
\left\{\begin{array}{l}
\dot{\tilde{z}}=F\left(z_{r}+\tilde{z}, x_{r}+\tilde{x}\right)-F\left(z_{r}, x_{r}\right), \\
\dot{\tilde{x}}=\mathcal{S} \tilde{x}+B \tilde{u}+f\left(z_{r}+\tilde{z}, x_{r}+\tilde{x}\right)-f\left(z_{r}, x_{r}\right)-\delta_{r},
\end{array}\right.
$$

where $B=(0, \ldots, 1)^{T}$ and $\delta_{r}=\left(\delta_{r, 1}, \ldots, \delta_{r, n}\right)$.

The objective is to find $\tilde{u}$ depending on the output $\tilde{x}_{1}=y-x_{r, 1}$ and on the state reference trajectory $\left(z_{r}, x_{r}\right)$ such that (15) holds.

\subsubsection{A high-gain domination approach}

The $\tilde{x}$ dynamics of the system (17) have the structure of a chain of integrators disturbed by nonlinear terms depending on the tracking error $(\tilde{x}, \tilde{z})$, the state reference trajectory $\left(x_{r}, z_{r}\right)$ and the approximation error $\delta_{r}$. This motivates us to use the domination approach introduced in [12] (see also [21]). In this context, the nonlinear functions (the $f_{i}$ 's) are not used in the design but considered as perturbations and the output feedback is designed on a dominating model which in this case is the chain of integrators. To ensure robustness to these nonlinearities, we employ high-gain techniques. This leads us to design the controller with the scaled coordinates :

$$
\tilde{\mathcal{X}}_{i}=L^{b+1-i} \tilde{x}_{i}
$$

where $L$ is the updated high-gain and $b$ is a positive real number satisfying (10). Compared to the scaled coordinates used in the high-gain approach in [12], we add an extra high-gain parameter $b$ which has been introduced in [19] and which allows gain adaptation.

\subsubsection{Homogeneous in the bi-limit output feedback for a chain of integrators}

Following the domination approach, we focus on the dominant part of system (17) in the scaled coordinate (18), i.e. a chain of integrators, with state $\mathfrak{X}=\left(\mathcal{X}_{1}, \ldots, \mathcal{X}_{n}\right)$ in $\mathbb{R}^{n}$ described by :

$$
\dot{\mathfrak{X}}=\mathcal{S} \mathfrak{X}+B u, \quad y=\mathcal{X}_{1} .
$$

To design the output feedback controller for system (19) we use the tools developed within the framework of homogeneity in the bi-limit, introduced in [2]. (See Appendix A for a brief summary.)

Selecting $\mathfrak{d}_{0}=0$ and with $\mathfrak{d}_{\infty}$ given by Assumption 2 , homogeneity in the bi-limit is obtained for system (19) with the weights $r_{0}=\left(r_{0,1}, \ldots, r_{0, n}\right)$ and $r_{\infty}=\left(r_{\infty, 1}, \ldots, r_{\infty, n}\right)$ as :

$$
r_{0, i}=1, \quad r_{\infty, i}=1-\mathfrak{d}_{\infty}(n-i) \quad, \quad i \in\{1, \ldots n\} .
$$

\footnotetext{
${ }^{8}$ Note that $r_{\infty, i}$ increases with $i$ and is in $(0,1]$.
} 
In [2] we have proposed an output feedback for system (19) given by :

$$
u=\phi(\hat{\mathfrak{X}}) \quad, \quad \dot{\hat{\mathfrak{X}}}=\mathcal{S} \hat{\mathfrak{X}}+B \phi(\hat{\mathfrak{X}})+K\left(\hat{\mathcal{X}}_{1}-\mathcal{X}_{1}\right)
$$

where $\hat{\mathfrak{X}}=\left(\hat{\mathcal{X}}_{1}, \ldots, \hat{\mathcal{X}}_{n}\right)$ is in $\mathbb{R}^{n}, K$ is a homogeneous in the bi-limit vector field with weights $r_{0}$ and $r_{\infty}$, and degrees 0 and $\mathfrak{d}_{\infty}$ and $\phi$ is a homogeneous in the bi-limit function with weights $r_{0}$ and $r_{\infty}$, and degrees 1 and $1+\mathfrak{d}_{\infty}$. Setting :

$$
E=\left(e_{1} \ldots, e_{n}\right)^{T}=\hat{\mathfrak{X}}-\mathfrak{X}
$$

the chain of integrators (19) with the controller (21) can be described by :

$$
\left\{\begin{array}{l}
\dot{\hat{\mathfrak{X}}}=\mathcal{S} \hat{\mathfrak{X}}+B \phi(\hat{\mathfrak{X}})+K\left(e_{1}\right) \\
\dot{E}=\mathcal{S} E+K\left(e_{1}\right) .
\end{array}\right.
$$

In [2], the design of $K$ and $\phi$ is performed recursively by following an observer / controller approach in such a way that there exists a homogeneous in the bi-limit Lyapunov function $U$ of degree $d_{U}$ satisfying, for some real number $c_{1}$,

$$
\begin{aligned}
\frac{\partial U}{\partial E}(\hat{\mathfrak{X}}, E)\left(\mathcal{S} E+K\left(e_{1}\right)\right)+\frac{\partial U}{\partial \mathfrak{X}}(\hat{\mathfrak{X}}, E)(\mathcal{S} \hat{\mathfrak{X}}+B \phi(\hat{\mathfrak{X}})+ & \left.K\left(e_{1}\right)\right) \leq \\
& -c_{1}\left(U(\hat{\mathfrak{X}}, E)+U(\hat{\mathfrak{X}}, E)^{\frac{d_{U}+\mathrm{d}_{\infty}}{d_{U}}}\right) .
\end{aligned}
$$

To combine this tool with dynamic scaling we need to establish a specific property on the Lyapunov function $U$. This property is a homogeneous in the bi-limit version of the one given in [19, equation (16)] or in [13, Lemma A1] and also used in the context of observer design in 四. Namely, given the diagonal matrix

$$
D=\operatorname{diag}(b, 1+b, \ldots, n-1+b)
$$

where $b$ is a positive real number satisfying (10), the function $\phi$ and the vector field $K$ are selected such that the Lyapunov function $U$ satisfies (23), and also :

$$
\frac{\partial U}{\partial E}(\hat{\mathfrak{X}}, E) D E+\frac{\partial U}{\partial \hat{\mathfrak{X}}}(\hat{\mathfrak{X}}, E) D \hat{\mathfrak{X}} \geq c_{2} U(\hat{\mathfrak{X}}, E),
$$

for some positive real number $c_{2}$.

Such a property can be obtained by modifying the recursive procedure given in [2] as claimed in the following statement the proof of which is omitted but can be found in [5].

Theorem 2 Let $d_{U}$ be a positive real number satisfying $d_{U} \geq 2 \max _{1 \leq j \leq n} r_{0, j}+\mathfrak{d}_{\infty}$. There exists a homogeneous in the bi-limit function $\phi: \mathbb{R}^{n} \rightarrow \mathbb{R}_{+}$with associated triples $\left(r_{0}, 1, \phi_{0}\right)$ and $\left(r_{\infty}, 1+\mathfrak{d}_{\infty}, \phi_{\infty}\right)$, a homogeneous in the bi-limit vector field $K: \mathbb{R} \rightarrow \mathbb{R}^{n}$, with associated triples $\left(r_{0}, \mathfrak{d}_{0}, K_{0}\right)$ and $\left(r_{\infty}, \mathfrak{d}_{\infty}, K_{\infty}\right)$ and a positive definite, proper and $C^{1}$ function $U: \mathbb{R}^{2 n} \rightarrow$ $\mathbb{R}_{+}$, homogeneous in the bi-limit with associated triples $\left(r_{0}, d_{U}, U_{0}\right)$ and $\left(r_{\infty}, d_{U}, U_{\infty}\right)$, such that the following holds.

1. The homogeneous approximating functions $U_{0}$ and $U_{\infty}$ are positive definite and proper and for all $j$ in $\{1, \ldots, n\}$, the functions $\frac{\partial U}{\partial e_{j}}$ and $\frac{\partial U}{\partial \hat{x}_{j}}$ are homogeneous in the bi-limit with approximating functions $\left(\frac{\partial U_{0}}{\partial e_{j}}, \frac{\partial U_{\infty}}{\partial e_{j}}\right)$ and $\left(\frac{\partial U_{0}}{\partial \hat{\mathcal{x}}_{j}}, \frac{\partial U_{\infty}}{\partial \hat{\mathcal{x}}_{j}}\right)$ respectively.

2. There exist two positive real numbers $c_{1}$ and $c_{2}$ such that (23) and (24) are satisfied. 
We choose $d_{U}$ satisfying also $\frac{d_{U}-1}{1+\mathfrak{d}_{\infty}}>q_{1}$, with $q_{1}$ given in Assumption 2 . In this way the functions $\mu_{i}^{q}$ are convex for all $q \geq \frac{d_{U}-1}{1+\mathfrak{d}_{\infty}}$. In what follows the parameters $d_{U}$ and $b$, the vector field $K$ and the function $\phi$ are assumed fixed, it remains to select the parameters $a_{1}, a_{2}$ and $a_{3}$ appearing in the high-gain updated law (11).

\subsection{Properties of the closed loop system}

\subsubsection{ISS property with respect to the $\delta_{r, i}$ 's}

The system (4) with the controller (8), (11), introduced in Section 2.2, can be fully described by the high-gain update law (11), the $\tilde{z}$ dynamics in (17) and the equations

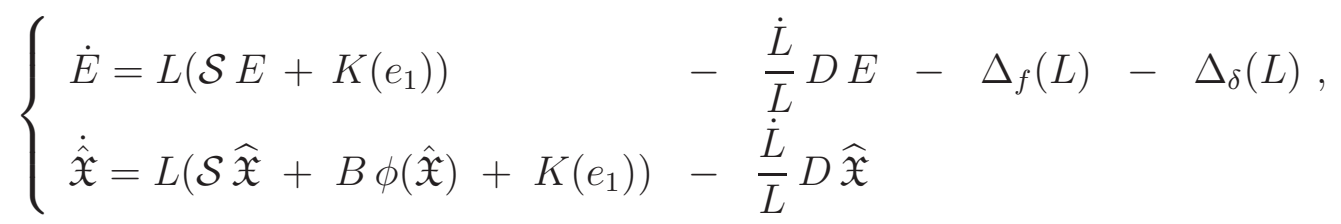

where $E=\left(e_{1}, \ldots, e_{n}\right)^{T}$ and $\hat{\mathfrak{X}}=\left(\hat{\mathcal{X}}_{1}, \ldots, \hat{\mathcal{X}}_{n}\right)^{T}$ are defined as :

$$
\hat{\mathfrak{X}}=\mathfrak{L}^{-1} \hat{x} \quad, \quad E=\mathfrak{L}^{-1}(\hat{x}-\tilde{x})
$$

and

$$
\Delta_{f}(L)=\mathfrak{L}^{-1}\left(f\left(z_{r}+\tilde{z}, x_{r}+\tilde{x}\right)-f\left(z_{r}, x_{r}\right)\right) \quad, \quad \Delta_{\delta}(L)=\mathfrak{L}^{-1}\left(\delta_{r, 1}, \ldots, \delta_{r, n}\right)
$$

are regarded as perturbations.

By inequality (23), the function $U$ obtained from Theorem 2 satisfies, along the solutions of system (25),

$$
\overparen{U(\hat{\mathfrak{X}}, E)} \leq-c_{1} L \theta(U(\hat{\mathfrak{X}}, E))+T_{D S}+T_{\text {Dist }},
$$

where $\theta$ is the function defined on $\mathbb{R}_{+}$as :

$$
\theta(s)=s+s^{\frac{\mathfrak{0} \infty+d_{U}}{d_{U}}},
$$

and

$$
\begin{aligned}
T_{D S} & =-\frac{\dot{L}}{L}\left(\frac{\partial U}{\partial \hat{\mathfrak{X}}}(\hat{\mathfrak{X}}, E) D \hat{\mathfrak{X}}+\frac{\partial U}{\partial E}(\hat{\mathfrak{X}}, E) D E\right), \\
T_{\text {Dist }} & =-\frac{\partial U}{\partial E}(\hat{\mathfrak{X}}, E)\left[\Delta_{f}(L)+\Delta_{\delta}(L)\right] .
\end{aligned}
$$

The above discussion and Assumption 2 yield the following result, the proof of which is given in Appendix B.2.

Lemma 1 There exist two positive real numbers $c_{3}$ and $c_{4}$ such that, for all sufficiently small strictly positive real numbers $a_{1}$ and sufficiently large real numbers $a_{2}$ and $a_{3}$, inequality (2X) becomes :

$$
\overparen{U(\hat{\mathfrak{X}}, E)} \leq-4 c_{3} L \theta(U(\hat{\mathfrak{X}}, E))+c_{4} L^{1-\epsilon} \sum_{i=1}^{n} \zeta_{i}\left(Y_{i}\right)+L c_{4} \sum_{i=1}^{n} \zeta_{i}\left(\frac{\delta_{r, i}}{L^{i+b}}\right)
$$

${ }^{9}$ Note that $\stackrel{\dot{\mathfrak{L}^{-1}}}{=}=-L^{-1} \dot{L} D \mathfrak{L}^{-1}$ 
where

$$
Y_{i}=L^{\epsilon-(i+b)} \mu_{i}\left(Z_{i}\left(z_{r}, \tilde{z}\right)\right)
$$

and the $\zeta_{i}$ are $C^{1}$, convex, strictly increasing and homogeneous in the bi-limit functions with weights 1 and $r_{\infty, i}+\mathfrak{d}_{\infty}$ and degrees $d_{U}$ and $d_{U}+\mathfrak{d}_{\infty}$, defined as

$$
\begin{aligned}
& \zeta_{i}(s)=\int_{0}^{s} \max \left\{|\sigma|^{d_{U}-1},|\sigma|^{\frac{d_{U}-r_{\infty, i}}{r_{\infty}+i+\infty}}\right\} d \sigma \quad, \quad \forall i \in\{1 \ldots, n-1\}, \\
& \zeta_{n}(s)=\int_{0}^{s} \min \left\{|\sigma|^{d_{U}-1},|\sigma|^{\frac{d_{U}-1}{1+\boldsymbol{1} \infty}}\right\} d \sigma .
\end{aligned}
$$

If the functions $Z_{i}$ 's were not present (via $Y_{i}$ ), inequality (29) would give readily an ISS property between $\frac{\delta_{r, i}}{L^{i+b}}$ and $(\hat{\mathfrak{X}}, E)$. To prove this claim note that the function

$$
s \in \mathbb{R}^{n} \mapsto\left(\sum_{i=1}^{n-1}\left|s_{i}\right|+\left|s_{i}\right|^{\frac{1}{r_{\infty}, i+\mathfrak{d} \infty}}+\frac{\left|s_{n}\right|}{1+\left|s_{n}\right|}\left(1+\left|s_{n}\right|^{\frac{1}{1+\mathfrak{d} \infty}}\right)\right)^{d_{U}}
$$

is positive definite, homogeneous in the bi-limit with weights 1 and $r_{\infty, i}+\mathfrak{d}_{\infty}$ and degrees $d_{U}$ and $d_{U}$ and its homogeneous approximating functions (i.e. $\sum_{i=1}^{n}\left|s_{i}\right|^{d_{U}}$ and $\sum_{i=1}^{n}\left|s_{i}\right|^{\frac{d_{U}}{r_{\infty}+i_{\infty}}}$ ) are positive definite. Furthermore, since the function $s \in \mathbb{R}^{n} \mapsto \theta^{-1}\left(\frac{c_{4}}{c_{3}} \sum_{i=1}^{n} \zeta_{i}\left(s_{i}\right)\right)$ is homogeneous in the bi-limit with the same weights and degree $\square$ by Claim A.3 in Appendix A, there exists a positive real number $c_{5}$ such that:

$$
\begin{aligned}
\theta^{-1}\left(\frac{c_{4}}{c_{3}} \sum_{i=1}^{n} \zeta_{i}\left(s_{i}\right)\right) & \leq c_{5}^{d_{U}}\left(\sum_{i=1}^{n-1}\left|s_{i}\right|+\left|s_{i}\right|^{\frac{1}{r_{\infty}, i+\mathrm{d} \infty}}+\frac{\left|s_{n}\right|}{1+\left|s_{n}\right|}\left(1+\left|s_{n}\right|^{\frac{1}{1+\boldsymbol{\jmath} \infty}}\right)\right)^{d_{U}}, \\
& \leq c_{5}^{d_{U}}\left(\sum_{i=1}^{n-1}\left|s_{i}\right|+\left|s_{i}\right|^{\frac{1}{r_{\infty, i}+\boldsymbol{\jmath} \infty}}+2\left|s_{n}\right|\right)^{d_{U}}
\end{aligned}
$$

Hence, without $Y_{i}$ in the inequality (29), we would have (see [22]) :

$$
\limsup _{t \rightarrow+\infty} U(\hat{\mathfrak{X}}, E(t)) \leq c_{5}^{d_{U}} \limsup _{t \rightarrow+\infty}\left[\sum_{i=1}^{n-1}\left(\frac{\left|\delta_{r, i}(t)\right|}{L(t)^{i+b}}\right)+\left(\frac{\left|\delta_{r, i}(t)\right|}{L(t)^{i+b}}\right)^{\frac{1}{r_{\infty, i}+\boldsymbol{\nabla} \infty}}+2 \frac{\left|\delta_{r, n}(t)\right|}{L(t)^{n+b}}\right]^{d_{U}}
$$

In addition, since the function $U$ is positive definite, homogeneous in the bi-limit with degrees $d_{U}$ and $d_{U}$ and its homogeneous approximating functions are positive definite, and since $r_{\infty, i}$ is smaller or equal to 1, Claim A.3 in Appendix A implies the existence of a positive real number $c_{13}$ such that:

$$
U(\mathfrak{X}, E) \geq c_{13}|\hat{\mathfrak{X}}-E|^{d_{U}}=c_{13}\left|\mathfrak{L}^{-1} \tilde{x}\right|^{d_{U}}
$$

which implies that (15) holds with $c_{r}=\frac{c_{5}}{c_{13}^{d_{U}}}$.

\footnotetext{
${ }^{10}$ Recall that $d_{U}-1 \geq \frac{d_{U}-r_{\infty, i}}{r_{\infty, i}}$ for all $i$ in $\{1, \ldots, n-1\}$ and $d_{U}-1 \geq \frac{d_{U}-1}{1+\mathfrak{d}_{\infty}}$.

$11 \theta: \mathbb{R}_{+} \rightarrow \mathbb{R}_{+}$defined in $(28)$ is a bijective, homogeneous in the bi-limit function, and satisfies all assumptions of [2, Proposition 2.11]. This implies homogeneity in the bi-limit of its inverse map $\theta^{-1}: \mathbb{R}_{+} \rightarrow \mathbb{R}_{+}$ with approximating homogeneous functions $s$ and $s^{\frac{d_{U}}{\mathrm{D}^{\infty}+d_{U}}}$. Then [2, Proposition 2.10] implies that the function $\theta^{-1}\left(\frac{c_{4}}{c_{3}} \sum_{i=1}^{n} \zeta_{i}\left(s_{i}\right)\right)$ is homogeneous in the bi-limit with degrees $d_{U}$ and $d_{U}$.
} 


\subsubsection{Small-gain arguments.}

To establish an inequality like (33) in the presence of $Z_{i}$ (or $Y_{i}$ ), we need a more advanced argument relying on a small gain theorem.

First of all, note that, Assumptions 1 and 3 yield the following result, the proof of which is in Appendix B.3.

Lemma 2 There exist two positive real numbers $c_{6}$ and $c_{7}$ such that, for all sufficiently small strictly positive real number $a_{1}$ and sufficiently large real numbers $a_{2}$ and $a_{3}$, we have, along the trajectories of the closed-loop system,

$$
\overparen{\zeta_{i}\left(Y_{i}\right)} \leq-c_{6} \zeta_{i}\left(Y_{i}\right)+c_{7} \bar{\Omega}\left(z_{r}, x_{r}\right) \theta(U(\hat{\mathfrak{X}}, E)), \quad \forall i\{1, \ldots, n\}
$$

Lemma 2 quantifies the ISS gain between $(\hat{\mathfrak{X}}, E)$ and $Y_{i}$, which, unfortunately, depends on $\left(x_{r}, z_{r}\right)$ (through the function $\bar{\Omega}$ ). Nevertheless, due to the special structure of the high-gain update law (11), an inequality similar to (33) can still be obtained.

Suppose that $a_{1}$ is sufficiently small and that $a_{2}$ and $a_{3}$ are sufficiently large such that Lemmas 1 and 12 apply. Then, inequality (35) yields, for all $0 \leq s \leq t$,

$$
\zeta_{i}\left(Y_{i}(t)\right) \leq \exp \left(c_{6}(s-t)\right) \zeta_{i}\left(Y_{i}(s)\right)+\int_{s}^{t} \exp \left(c_{6}(r-t)\right) c_{7} \bar{\Omega}(r) \theta(U(r)) d r,
$$

where we have used the compact notation :

$$
U(t)=U(\hat{\mathfrak{X}}(t), E(t)), Y_{i}(t)=L(t)^{\epsilon-(i+b)} \mu_{i}\left(Z_{i}\left(z_{r}(t), \tilde{z}(t)\right)\right), \bar{\Omega}(t)=\bar{\Omega}\left(z_{r}(t), x_{r}(t)\right) .
$$

The inequality above, together with definition (30) and inequality (29) divided by $L^{1-\epsilon}$, yields :

$$
\begin{aligned}
\frac{\dot{U(t)}}{L(t)^{1-\epsilon}} \leq-4 c_{3} L(t)^{\epsilon} \theta(U(t)) & +L(t)^{\epsilon} c_{4} \sum_{i=1}^{n} \zeta_{i}\left(\frac{\delta_{r, i}(t)}{L(t)^{i+b}}\right) \\
& +c_{4} \exp \left(c_{6}(s-t)\right) \sum_{i=1}^{n} \zeta_{i}\left(L(s)^{\epsilon-(i+b)}\left(\mu_{i}\left(Z_{i}(s)\right)\right)\right. \\
& +n c_{4} c_{7} \int_{s}^{t} \exp \left(c_{6}(r-t)\right)[\bar{\Omega}(r) \theta(U(r))] d r
\end{aligned}
$$

where :

$$
Z_{i}(t)=Z_{i}\left(z_{r}(t), \tilde{z}(t)\right)
$$

This yields :

$$
\begin{aligned}
\frac{\dot{U(t)}}{L(t)^{1-\epsilon}} \leq-4 c_{3} L(t)^{\epsilon} \theta( & U(t))+L(t)^{\epsilon} c_{4} \sum_{i=1}^{n} \zeta_{i}\left(\frac{\delta_{r, i}(t)}{L(t)^{i+b}}\right) \\
& +c_{4} \exp \left(c_{6}(s-t)\right) \sum_{i=1}^{n} \zeta_{i}\left(L(s)^{\epsilon-(i+b)}\left(\mu_{i}\left(Z_{i}(s)\right)\right)\right. \\
& +n c_{4} c_{7} \sup _{[s, t]}\left\{\exp \left(\frac{c_{6}}{2}(r-t)\right) \theta(U(r))\right\} \int_{s}^{t} \exp \left(\frac{c_{6}}{2}(r-t)\right) \bar{\Omega}(r) d r
\end{aligned}
$$


Now, assume for the time being that $L$ satisfies

$$
L(t)^{\epsilon} \geq \frac{1}{c_{3}}\left[n c_{4} c_{7} \int_{s}^{t} \exp \left(\frac{c_{6}}{2}(r-t)\right) \bar{\Omega}(r) d r+1\right] \quad \forall t \geq 0 .
$$

In this case (37) becomes, for all $s \leq t$,

$$
\begin{aligned}
\frac{\dot{U(t)}}{L(t)^{1-\epsilon} \leq-4 c_{3} L(t)^{\epsilon} \theta(U(t))}+L(t)^{\epsilon} c_{4} \sum_{i=1}^{n} \zeta_{i}\left(\frac{\delta_{r, i}(t)}{L(t)^{i+b}}\right) & \\
& +c_{4} \exp \left(c_{6}(s-t)\right) \sum_{i=1}^{n} \zeta_{i}\left(L(s)^{\epsilon-(i+b)}\left(\mu_{i}\left(Z_{i}(s)\right)\right)\right. \\
& +\left[c_{3} L(t)^{\epsilon}-1\right] \exp \left(-\frac{c_{6}}{2} t\right) \sup _{[s, t]}\left\{\exp \left(\frac{c_{6}}{2} r\right) \theta(U(r))\right\} \\
\leq \quad-\left[4 c_{3} L(t)^{\epsilon}+1\right] \theta(U(t)) & +L(t)^{\epsilon} c_{4} \sum_{i=1}^{n} \zeta_{i}\left(\frac{\delta_{r, i}(t)}{L(t)^{i+b}}\right) \\
& +c_{4} \exp \left(c_{6}(s-t)\right) \sum_{i=1}^{n} \zeta_{i}\left(L(s)^{\epsilon-(i+b)}\left(\mu_{i}\left(Z_{i}(s)\right)\right)\right. \\
& +c_{3} L(t)^{\epsilon} \exp \left(-\frac{c_{6}}{2} t\right) \sup _{[s, t]}\left\{\exp \left(\frac{c_{6}}{2} r\right) \theta(U(r))\right\}
\end{aligned}
$$

Since the functions $\delta$ and $L$ are upper bounded on $\mathbb{R}_{+}$and $L(0)>a_{2}^{\frac{1}{\epsilon}} \geq 1$, there exist $\partial_{m}$ and $L_{m}$ that satisfy :

$$
\partial_{m} \geq \sum_{i=1}^{n} \zeta_{i}\left(\frac{\delta_{r, i}(t)}{L(t)^{i+b}}\right), \quad L_{m} \geq L(t) \geq a_{2}^{\frac{1}{\epsilon}} \geq 1, \quad \forall t \geq 0 .
$$

Then, given the initial conditions $L(0)$ and $Z_{i}(0)$, we can find a sufficiently large positive real number $U_{m}$, strictly larger than $U(0)$, and satisfying, for all $i$,

$$
\theta\left(U_{m}\right) \geq c_{4} \sum_{i=1}^{n} \zeta_{i}\left(L(0)^{\epsilon-(i+b)}\left(\mu_{i}\left(Z_{i}(0)\right)\right)+c_{4} L_{m}^{\epsilon} \partial_{m}\right.
$$

Suppose there exists a time $t_{m}$ in the positive time domain of existence of the solution such that $U\left(t_{m}\right)=U_{m}$ and $U(r)<U_{m}$ for all $r$ in $\left[0, t_{m}\right)$. This implies

$$
\sup _{\left[0, t_{m}\right]}\left\{\exp \left(\frac{c_{6}}{2} r\right) \theta(U(r))\right\}=\exp \left(\frac{c_{6}}{2} t_{m}\right) \theta\left(U\left(t_{m}\right)\right) \text {. }
$$

On the other hand, by (41), setting $s=0$ and $t=t_{m}$ in (39) yields

$$
\frac{\overparen{U\left(t_{m}\right)}}{L\left(t_{m}\right)^{1-\epsilon}} \leq-3 c_{3} L\left(t_{m}\right)^{\epsilon} \theta\left(U\left(t_{m}\right)\right)<0 .
$$

This contradicts the definition of $t_{m}$. Consequently, $U_{m}$ upper bounds the function $t \mapsto U(t)$ in the positive time domain of existence of the solution. Since $t \mapsto L(t)$ is bounded, the same

\footnotetext{
${ }^{12}$ This property will be established at the end of the proof.
} 
holds for $t \mapsto(\hat{x}(t), \tilde{x}(t))$ and therefore also for $t \mapsto \gamma_{i}\left(z_{r}(t), x_{r}(t), \tilde{x}(t)\right)$. Then, by integration, we obtain from Assumption 1.2 that, for all $i$, the function $t \mapsto Z_{i}\left(z_{r}(t), \tilde{z}(t)\right)$ is bounded on the positive time domain of existence of the solution. Hence, by Assumption 1.1, the same holds for $t \mapsto \tilde{z}(t)$. This implies that the positive time domain of existence is $[0,+\infty)$ and that the closed loop solution is bounded on this interval.

It remains to establish (15). Let $\underline{U}$ be any positive real number satisfying

$$
\underline{U}>c_{5}^{d_{U}} \limsup _{t \rightarrow+\infty}\left(\sum_{i=1}^{n-1} \frac{\left|\delta_{r, i}(t)\right|}{L(t)^{i+b}}+\left(\frac{\left|\delta_{r, i}(t)\right|}{L(t)^{i+b}}\right)^{\frac{1}{r_{\infty, i}+\boldsymbol{\nu} \infty}}+2 \frac{\left|\delta_{r, n}(t)\right|}{L(t)^{b+n}}\right)^{d_{U}},
$$

with $c_{5}$ given in (32). By definition of limsup, there exists $t_{l}$ such that

$$
\underline{U} \geq c_{5}^{d_{U}}\left(\sum_{i=1}^{n-1}\left(\frac{\left|\delta_{r, i}(t)\right|}{L(t)^{i+b}}\right)+\left(\frac{\left|\delta_{r, i}(t)\right|}{L(t)^{i+b}}\right)^{\frac{1}{r_{\infty, i}+\mathrm{d} \infty}}+2 \frac{\left|\delta_{r, n}(t)\right|}{L(t)^{b+n}}\right)^{d_{U}} \quad, \quad \forall t \geq t_{l} .
$$

Furthermore, using (32), we obtain :

$$
L(t)^{\epsilon} c_{4} \sum_{i=1}^{n} \zeta_{i}\left(\frac{\delta_{r, i}(t)}{L(t)^{i+b}}\right) \leq c_{3} L(t)^{\epsilon} \theta(\underline{U}) \quad, \quad \forall t \geq t_{l} .
$$

Let $Z_{i, m}$ be a bound for the function $t \mapsto Z_{i}(t)$ and :

$$
t_{k}=\frac{1}{c_{6}} \ln \left(\frac{c_{4} \sum_{i=1}^{n} \zeta_{i}\left(a_{2}^{1-\frac{i+b}{\epsilon}} \mu_{i}\left(Z_{i, m}\right)\right)}{c_{3} a_{2} \theta(\underline{U})}\right) .
$$

By (40), we have :

$$
c_{4} \exp \left(c_{6}(s-t)\right) \sum_{i=1}^{n} \zeta_{i}\left(L(s)^{\epsilon-(i+b)}\left(\mu_{i}\left(Z_{i}(s)\right)\right) \leq c_{3} L(t)^{\epsilon} \theta(\underline{U}) \quad, \quad \forall t \geq s+t_{k} .\right.
$$

Define now

$$
\mathcal{U}(s)=\sup _{r \geq s} U(r)
$$

and assume that we have :

$$
\mathcal{U}(s) \geq \underline{U} \quad \forall s \geq t_{l} .
$$

Then, by (44) and (45), (39) with $s \geq t_{l}$, gives :

$$
\frac{\overparen{U(t)}}{L(t)^{1-\epsilon}} \leq-\left[4 c_{3} L(t)^{\epsilon}+1\right] \theta(U(t))+3 c_{3} L(t)^{\epsilon} \theta(\mathcal{U}(s)) \quad \forall t \geq s+t_{k}
$$

So, for each time $t \geq s+t_{k}$ for which we have $\theta(U(t)) \geq \frac{3}{4} \theta(\mathcal{U}(s))$, we obtain :

$$
\overbrace{U(t)}^{\cdot}<-\theta(U(t))
$$

It follows from the proof of [23, Theorem 1] that we have :

$$
\limsup _{t \rightarrow+\infty} \theta(U(t)) \leq \frac{3}{4} \theta(\mathcal{U}(s))
$$


But the definition (28) of $\theta$ gives :

$$
\theta(a) \leq \rho \theta(b) \quad \Rightarrow \quad a \leq \rho^{\frac{d_{U}}{\rho_{0}+d_{U}}} b \quad \forall a, b \geq 0, \quad \forall \rho \in[0,1] .
$$

This yields :

$$
\limsup _{t \rightarrow+\infty} U(t) \leq\left(\frac{3}{4}\right)^{\frac{d_{U}}{\mathrm{~d}_{\infty}+d_{U}}} \mathcal{U}(s)
$$

and this for all $s \geq t_{l}$. By taking the limit for $s$ going to infinity, we get

$$
\limsup _{t \rightarrow+\infty} U(t) \leq\left(\frac{3}{4}\right)^{\frac{d_{U}}{\mathrm{~d}_{\infty}+d_{U}}} \lim \sup _{t \rightarrow+\infty} U(t)
$$

and therefore $\lim \sup _{t \rightarrow+\infty} U(t)=0$, which contradicts (46). We conclude that, for each $\underline{U}$ satisfying (42), there exists $s$ satisfying :

$$
\mathcal{U}(s)=\sup _{r \geq s} U(r)<\underline{U}
$$

from which (15) follows.

To complete the proof it remains to show that the property (38) is satisfied. By letting

$$
M=L^{\epsilon},
$$

(11) gives :

$$
\dot{M}=-\epsilon a_{1} M+\epsilon M \max \left\{0, a_{1}\left(a_{2}+1-M\right)+a_{3} \bar{\Omega}\left(z_{r}, x_{r}\right)\right\} .
$$

Since $M \geq a_{2} \geq 1$, when :

$$
a_{1}\left(a_{2}+1\right)+a_{3} \bar{\Omega}\left(z_{r}, x_{r}\right) \geq a_{1} M
$$

we get :

$$
\begin{aligned}
\dot{M} & \geq-\epsilon a_{1} M+\epsilon\left[a_{1}\left(a_{2}+1-M\right)+a_{3} \bar{\Omega}\left(z_{r}, x_{r}\right)\right] \\
& \geq-2 \epsilon a_{1} M+\epsilon\left[a_{1}\left(a_{2}+1\right)+a_{3} \bar{\Omega}\left(z_{r}, x_{r}\right)\right] .
\end{aligned}
$$

and, when :

$$
a_{1}\left(a_{2}+1\right)+a_{3} \bar{\Omega}\left(z_{r}, x_{r}\right)<a_{1} M
$$

we get :

$$
\begin{aligned}
\dot{M} & =-2 \epsilon a_{1} M+\epsilon a_{1} M, \\
& \geq-2 \epsilon a_{1} M+\epsilon\left[a_{1}\left(a_{2}+1\right)+a_{3} \bar{\Omega}\left(z_{r}, x_{r}\right)\right] .
\end{aligned}
$$

By integration this yields :

$$
M(t) \geq \exp \left(-2 \epsilon a_{1} t\right)\left(M(0)-\frac{a_{2}+1}{2}\right)+\varepsilon a_{3} \int_{0}^{t} \exp \left(2 \epsilon a_{1}(r-t)\right) \Omega\left(z_{r}(r), x_{r}(r)\right) d r+\frac{a_{2}+1}{2} .
$$

Picking $L(0)^{\epsilon}=M(0) \geq a_{2}$, the inequality (38) holds provided $a_{1}$ is chosen sufficiently small and $a_{2}$ and $a_{3}$ sufficiently large. 


\section{Conclusion}

We have solved a tracking problem by output feedback for minimum phase non-linear systems which admit globally a strict normal form. Unlike most existing results, we allow nonlinearities in the model satisfying a polynomial type growth in the unmeasured state components. In particular, the result obtained generalizes the one obtained in [8] since asymptotic tracking may be obtained without knowing an upper-bound on the reference trajectory.

This has been achieved by exploiting the tools of domination, homogeneity in the bi-limit, dynamic scaling and a novel time varying small gain argument.

\section{References}

[1] V. Andrieu, L. Praly, A Unifying point of view on output feedback designs. Submitted to Automatica.

[2] V. Andrieu, L. Praly, A. Astolfi, Homogeneous approximation, Recursive observer design and Output feedback. SIAM J. Control and Optimization, Vol.47, No.4, pp. 1814-1850, 2008 .

[3] V. Andrieu, L. Praly, A. Astolfi, Trajectory tracking by output feedback for some non linear systems, Proc. 46th IEEE CDC, San Diego, 2007.

[4] V. Andrieu, L. Praly, A. Astolfi, High gain observers with updated high-gain and homogeneous correction terms. To appear in Automatica

[5] V. Andrieu, L. Praly, A. Astolfi, An output feedback for a chain of integrator suitable for the use of dynamic scaling. HAL, http://hal.archives-ouvertes.fr/hal-00282708/fr/.

[6] D. Angeli, A Lyapunov approach to incremental stability. IEEE Transactions on Automatic Control, 47, pp. 410-422, 2002.

[7] Z. Chen, J. Huang, Global robust servomechanism problem for uncertain lower triangular nonlinear systems by output feedback control. Proc. of the 43 rd IEEE CDC, Bahamas, 2004 .

[8] Q. Gong, C. Qian, Global practical tracking of a class of nonlinear systems by output feedback. Automatica, Vol. 43, pp. 184-189, 2007.

[9] J. Grizzle, M. Di Benedetto, F. Lamnabhi-Lagarrigue, Necessary Conditions for Asymptotic Tracking in Nonlinear Systems. IEEE Transactions on Automatic Control, Vol. 39, No. 9, September 1994.

[10] R. Hirschorn, Invertibility of nonlinear control systems. SIAM J. Control and Optimization, Vol. 17, No. 2, pp. 289-297, 1979.

[11] A. Isidori, Nonlinear control systems: an introduction. Springer-Verlag New York, Inc. New York, NY, USA, 1989.

[12] H. Khalil, A. Saberi, Adaptive stabilization of a class of nonlinear systems using high-gain feedback. IEEE Transactions on Automatic Control, Vol. 32, No. 11, pp. 1031-1035, 1987. 
[13] P. Krishnamurthy, F. Khorrami, and R. S. Chandra, Global high-gain based observer and backstepping controller for generalized output-feedback canonical form. IEEE Transactions on Automatic Control, vol. 48, No. 12, pp. 2277-2284, 2003.

[14] P. Krishnamurthy, F. Khorrami, Dynamic high-gain scaling : state and output feedback with application to systems with ISS appended dynamics driven by all states. IEEE Transactions on Automatic Control, Vol. 49, No. 12, 2004.

[15] P. Krishnamurthy, F. Khorrami and Z. P. Jiang, Global output feedback tracking for nonlinear systems in generalized output-feedback canonical form. IEEE Transactions on Automatic Control, Vol.47, no.5, pp.814-819, 2002.

[16] M. Krstić, I. Kanellakopoulos, P. Kokotović, Nonlinear and adaptive control design. John Wiley $\&$ Sons, New York, 1995.

[17] F. Mazenc, L. Praly, and W. P. Dayawansa, Global stabilization by output feedback : Examples and Counter-Examples. Systems $\&$ Control Letters, Vol. 23, pp. 119-125, 1994.

[18] R. Marino, P. Tomei, Nonlinear control design. Geometric, adaptive, robust. Prentice Hall, (1995).

[19] L. Praly, Asymptotic stabilization via output feedback for lower triangular systems with output dependent incremental rate. IEEE Transactions on Automatic Control, Vol. 48, N. 6, 2003.

[20] L. Praly, Z-P Jiang, Further Results on Robust Semiglobal Stabilization with Dynamic Input Uncertainties, Proc. of the 37th IEEE CDC, Tampa, 1998.

[21] C. Qian, W. Lin, Output feedback control of a class of nonlinear systems : a nonseparation principle paradigm. IEEE Transactions on Automatic Control, Vol.47, 10, pp. 1710-1079, 2001.

[22] E.D. Sontag. Input to state stability: Basic concepts and results. In P. Nistri and G. Stefani, Editors, Nonlinear and Optimal Control Theory, pp. 163-220. Springer-Verlag, Berlin, 2006.

[23] E.D. Sontag. Smooth stabilization implies coprime factorization. IEEE Transactions on Automatic Control, Vol. 34, N. 4, pp. 435-443, 1989.

[24] M. Van Nieuwstadt, R. Murray Real-time trajectory generation for differentially flat systems, International Journal of Robust and Nonlinear Control, Vol. 8, No. 11, pp 9951020, 1998.

\section{A Homogeneity in the bi-limit}

For details on the notion of homogeneity in the bi-limit the reader is referred to [2]. For completeness, we recall the definition and state the main properties used in the paper.

Given a weight $r=\left(r_{1}, \ldots, r_{n}\right)$ in $\left(\mathbb{R}_{+} /\{0\}\right)^{n}$, we define the dilation of a vector $x$ in $\mathbb{R}^{n}$ as

$$
\lambda^{r} \diamond x=\left(\lambda^{r_{1}} x_{1}, \ldots, \lambda^{r_{n}} x_{n}\right)^{T} \text {. }
$$




\section{Definition 1 (Homogeneity in the 0-limit)}

- A continuous function $\phi: \mathbb{R}^{n} \rightarrow \mathbb{R}$ is said homogeneous in the 0-limit with associated triple $\left(r_{0}, d_{0}, \phi_{0}\right)$, where $r_{0}$ in $\left(\mathbb{R}_{+} /\{0\}\right)^{n}$ is the weight $\mathbb{T}^{\mathbb{3}}$, $d_{0}$ in $\mathbb{R}_{+}$the degree and $\phi_{0}: \mathbb{R}^{n} \rightarrow \mathbb{R}$ the approximating function, respectively, if $\phi_{0}$ is continuous and not identically zero and, for each compact set $C$ in $\mathbb{R}^{n} \backslash\{0\}$ and each $\varepsilon>0$, there exists $\lambda^{*}$ such that we have:

$$
\max _{x \in C}\left|\frac{\phi\left(\lambda^{r_{0}} \diamond x\right)}{\lambda^{d_{0}}}-\phi_{0}(x)\right| \leq \varepsilon \quad \forall \lambda \in\left(0, \lambda^{*}\right]
$$

- A vector field $f=\sum_{i=1}^{n} f_{i} \frac{\partial}{\partial x_{i}}$ is said homogeneous in the 0-limit with associated triple $\left(r_{0}, \mathfrak{d}_{0}, f_{0}\right)$, where $f_{0}=\sum_{i=1}^{n} f_{0, i} \frac{\partial}{\partial x_{i}}$, if, for each $i$ in $\{1, \ldots, n\}$, the function $f_{i}$ is homogeneous in the 0-limit with associated triple $\left(r_{0}, \mathfrak{d}_{0}+r_{0, i}, f_{0, i}\right)$ 四.

\section{Definition 2 (Homogeneity in the $\infty$-limit)}

- A continuous function $\phi: \mathbb{R}^{n} \rightarrow \mathbb{R}$ is said homogeneous in the $\infty$-limit with associated triple $\left(r_{\infty}, d_{\infty}, \phi_{\infty}\right)$ where $r_{\infty}$ in $\left(\mathbb{R}_{+} /\{0\}\right)^{n}$ is the weight, $d_{\infty}$ in $\mathbb{R}_{+}$the degree and $\phi_{\infty}: \mathbb{R}^{n} \rightarrow \mathbb{R}$ the approximating function, respectively, if $\phi_{\infty}$ is continuous and not identically zero and, for each compact set $C$ in $\mathbb{R}^{n} \backslash\{0\}$ and each $\varepsilon>0$, there exists $\lambda^{*}$ such that we have:

$$
\max _{x \in C}\left|\frac{\phi\left(\lambda^{r_{\infty}} \diamond x\right)}{\lambda^{d_{\infty}}}-\phi_{\infty}(x)\right| \leq \varepsilon \quad \forall \lambda \in\left[\lambda^{*},+\infty\right)
$$

- A vector field $f=\sum_{i=1}^{n} f_{i} \frac{\partial}{\partial x_{i}}$ is said homogeneous in the $\infty$-limit with associated triple $\left(r_{\infty}, \mathfrak{d}_{\infty}, f_{\infty}\right)$, with $f_{\infty}=\sum_{i=1}^{n} f_{\infty, i} \frac{\partial}{\partial x_{i}}$, if, for each $i$ in $\{1, \ldots, n\}$, the function $f_{i}$ is homogeneous in the $\infty$-limit with associated triple $\left(r_{\infty}, \mathfrak{d}_{\infty}+r_{\infty, i}, f_{\infty, i}\right)$.

\section{Definition 3 (Homogeneity in the bi-limit)}

A continuous function $\phi: \mathbb{R}^{n} \rightarrow \mathbb{R}$ (or a vector field $f$ ) is said homogeneous in the bi-limit if it is homogeneous in the 0-limit and homogeneous in the $\infty$-limit.

We now recall some properties of homogeneous in the bi-limit functions. Let $\eta$ and $\gamma$ be two continuous homogeneous in the bi-limit functions with weights $r_{0}, r_{\infty}$, degrees $d_{\eta, 0}, d_{\eta, \infty}$ and $d_{\gamma, 0}, d_{\gamma, \infty}$, and continuous approximating functions $\eta_{0}, \eta_{\infty}, \gamma_{0}, \gamma_{\infty}$.

Claim A.1. The function $x \mapsto \eta(x) \gamma(x)$ is homogeneous in the bi-limit with associated triples $\left(r_{0}, d_{\eta, 0}+d_{\gamma, 0}, \eta_{0} \gamma_{0}\right)$ and $\left(r_{\infty}, d_{\eta, \infty}+d_{\gamma, \infty}, \eta_{\infty} \gamma_{\infty}\right)$.

Claim A.2. If the degrees satisfy $d_{\eta, 0} \geq d_{\gamma, 0}$ and $d_{\eta, \infty} \leq d_{\gamma, \infty}$, and $\gamma(x) \geq 0$, and we have the following implications for all non-zero $x$ in $\mathbb{R}^{n}$ :

\footnotetext{
${ }^{13}$ If $x$ is partitioned as $\left(x_{a}, x_{b}\right)$, we use $\left(r_{0 a}, r_{0 b}\right)$ (respectively $\left.\left(r_{\infty a}, r_{\infty b}\right)\right)$ to denote the weights of $x_{a}$ and $x_{b}$.

${ }^{14}$ In the case of a vector field the degree $\mathfrak{d}_{0}$ can be negative as long as $\mathfrak{d}_{0}+r_{0, i} \geq 0$, for all $1 \leq i \leq n$.
} 


$$
\begin{array}{rlll}
\gamma(x)=0 & \Rightarrow & \eta(x)<0, \\
\gamma_{0}(x)=0 & \Rightarrow & & \eta_{0}(x)<0, \\
\gamma_{\infty}(x)=0 & \Rightarrow & & \eta_{\infty}(x)<0,
\end{array}
$$

then there exists a real number $k^{*}$ such that, for all $k \geq k^{*}$, and for all non-zero $x$ in $\mathbb{R}^{n}$ :

$$
\eta(x)<k \gamma(x), \eta_{0}(x)<k \gamma_{0}(x), \eta_{\infty}(x)<k \gamma_{\infty}(x) .
$$

Claim A.3. If the degrees satisfy $d_{\eta, 0} \geq d_{\gamma, 0}$ and $d_{\eta, \infty} \leq d_{\gamma, \infty}$ and the functions $\gamma, \gamma_{0}$ and $\gamma_{\infty}$ are positive definite then there exists a positive real number $c$ satisfying $\eta(x) \leq c \gamma(x)$ for all $x$ in $\mathbb{R}^{n}$.

\section{B Technical proofs}

\section{B.1 Proof of inequalities (13) and (14)}

For any $t_{0} \geq 1$, let

$$
\bar{L}_{t_{0}}=\left(a_{2}+\frac{1}{t_{0}}+\frac{a_{3}}{a_{1}} \sup _{s \geq t_{0}} \bar{\Omega}(s)\right)^{\frac{1}{\epsilon}} .
$$

Then from (11)

$$
\dot{L}(t) \leq-a_{1} L(t)+L(t) \max \left\{0, a_{1}\left(1-\frac{1}{t_{0}}+\bar{L}_{t_{0}}^{\epsilon}-L(t)^{\epsilon}\right)\right\} \quad \forall t \geq t_{0} .
$$

It follows that

$$
\dot{L}(t) \leq-\frac{a_{1}}{t_{0}} L(t) \quad \forall t \geq t_{0} \geq 1: L(t) \geq \bar{L}_{t_{0}} .
$$

From the proof of [23, Theorem 1], this yields :

$$
\limsup _{t \rightarrow+\infty} L(t) \leq \bar{L}_{t_{0}}
$$

As a result inequality (13) follows letting $t_{0}$ go to $+\infty$.

Similarly, let

$$
\underline{L}_{t_{0}}=\left(a_{2}-\frac{1}{t_{0}}+\frac{a_{3}}{a_{1}} \inf _{s \geq t_{0}} \bar{\Omega}(s)\right)^{\frac{1}{\epsilon}} \text {. }
$$

This gives

$$
\overparen{\underline{L}_{t_{0}}-L(t)} \leq-\frac{a_{1}}{t_{0}} L(t) \leq-\frac{a_{4}}{t_{0}}\left(\underline{L}_{t_{0}}-L(t)\right) \quad \forall t \geq t_{0}: \underline{L}_{t_{0}} \geq L(t) \quad\left(\geq a_{2}^{\frac{1}{\epsilon}}\right)
$$

where :

$$
a_{4}=\frac{a_{1} a_{2}^{\frac{1}{\epsilon}}}{\underline{L}_{t_{0}}-a_{2}^{\frac{1}{\epsilon}}} .
$$

It follows that

$$
\liminf _{t \rightarrow+\infty} L(t) \geq \underline{L}_{t_{0}},
$$

from which (14) follows letting $t_{0}$ go to $+\infty$. 


\section{B.2 Proof of Lemma 1}

This proof is composed of two steps. We first give a bound on $T_{D S}$ and then on $T_{D i s t}$.

Bound on the term $T_{D S}$. Equation (24), and the expression of $\dot{L}$ in (11), give :

$$
T_{D S} \leq-\left[a_{1}\left(a_{2}-L^{\epsilon}\right)+a_{3} \bar{\Omega}\left(z_{r}, x_{r}\right)\right]\left(\frac{\partial U}{\partial \hat{\mathfrak{X}}}(\hat{\mathfrak{X}}, E) D \hat{\mathfrak{X}}+\frac{\partial U}{\partial E}(\hat{\mathfrak{X}}, E) D E\right) .
$$

From Theorem 2, point 1), for each $i$ in $\{1, \ldots, n\}$ the functions $\frac{\partial U}{\partial e_{i}}(\hat{\mathfrak{X}}, E)$ and $\frac{\partial U}{\partial \hat{\mathcal{X}}_{i}}(\hat{\mathfrak{X}}, E)$ are homogeneous in the bi-limit with the same weights $\left(r_{0}, r_{0}\right)$ and $\left(r_{\infty}, r_{\infty}\right)$ and degrees $d_{U}-1$ and $d_{U}-r_{\infty, i}$. Hence, by Claim A.1 in Appendix @, the function $(\hat{\mathfrak{X}}, E) \mapsto \frac{\partial U}{\partial \hat{\mathfrak{X}}}(\hat{\mathfrak{X}}, E) D \hat{\mathfrak{X}}+$ $\frac{\partial U}{\partial E}(\hat{\mathfrak{X}}, E) D E$ is homogeneous in the bi-limit with weights $\left(r_{0}, r_{0}\right)$ and $\left(r_{\infty}, r_{\infty}\right)$ and degrees $d_{U}$ and $d_{U}$. Hence Claim A.3 yields a positive real number $c_{11}$ such that:

$$
\frac{\partial U}{\partial \hat{\mathfrak{X}}}(\hat{\mathfrak{X}}, E) D \hat{\mathfrak{X}}+\frac{\partial U}{\partial E}(\hat{\mathfrak{X}}, E) D E \leq c_{11} U(\hat{\mathfrak{X}}, E) .
$$

Finally, using (24) once again and since $a_{2}^{\frac{1}{\epsilon}} \leq L^{\epsilon} \leq L$, we get

$$
T_{D S} \leq-c_{2} a_{3} \bar{\Omega}\left(z_{r}, x_{r}\right) U(\hat{\mathfrak{X}}, E)+c_{11} a_{1} L U(\hat{\mathfrak{X}}, E) .
$$

Bound on the term $T_{\text {Dist }}$. By Assumption 2, (9), (26) and (20), we have, for all $i$,

$$
\begin{aligned}
& \left|\left(\Delta_{f}(L)\right)_{i}\right| \\
& \quad=L^{1-i-b}\left|f_{i}\left(z_{r}+\tilde{z}, x_{r}+\tilde{x}\right)-f_{i}\left(z_{r}, x_{r}\right)\right| \\
& \quad \leq \Omega\left(z_{r}, x_{r}\right) \sum_{j=1}^{i} L^{j-i}\left|\hat{\mathcal{X}}_{j}-e_{j}\right|+c_{\infty} L^{1-i-b} \sum_{j=1}^{i}\left|L^{b+j-1}\left(\hat{\mathcal{X}}_{j}-e_{j}\right)\right|^{\frac{r_{\infty, i}+\mathfrak{d} \infty}{r_{\infty, j}}}+L^{1-i-b} \mu_{i}\left(Z_{i}\right) .
\end{aligned}
$$

Inequality (12) implies that, for all $L \geq a_{2}^{\frac{1}{\epsilon}} \geq 1$,

$$
\frac{1}{a_{2}} \geq L^{-\epsilon} \geq L^{(b+j-1) \frac{r_{\infty, i}+\mathfrak{d}_{\infty}}{r_{\infty, j}}-i-b}
$$

Consequently, for all $(\hat{\mathfrak{X}}, E)$ in $\mathbb{R}^{2 n}$ and $L \geq a_{2}^{\frac{1}{\epsilon}} \geq 1$,

$$
\left|\left(\Delta_{f}(L)\right)_{i}\right| \leq \Omega\left(z_{r}, x_{r}\right) \sum_{j=1}^{i}\left|\hat{\mathcal{X}}_{j}-e_{j}\right|+\frac{c_{\infty}}{a_{2}} L \sum_{j=1}^{i}\left|\hat{\mathcal{X}}_{j}-e_{j}\right|^{\frac{r_{\infty, i}+\mathrm{D} \infty}{r_{\infty}, j}}+L^{1-i-b} \mu_{i}\left(Z_{i}\right) .
$$

On the other hand, the functions $\left|\frac{\partial U}{\partial e_{i}}(E)\right|\left|\hat{\mathcal{X}}_{j}-e_{j}\right|$ and $\left|\frac{\partial U}{\partial e_{i}}(E)\right|\left|\hat{\mathcal{X}}_{j}-e_{j}\right|^{\frac{r_{\infty, i}+\mathrm{d}_{\infty}}{r_{\infty, j}}}$ are homogeneous in the bi-limit with weights $\left(r_{0}, r_{0}\right)$ and $\left(r_{\infty}, r_{\infty}\right)$ and degrees $d_{U}$ and $d_{U}+r_{\infty, j}-r_{\infty, i}(\leq$ $\left.d_{U}\right)$ and, respectively, $d_{U}-1+\frac{r_{\infty, i}+\mathfrak{d}_{\infty}}{r_{\infty, j}}\left(\geq d_{U}\right)$ and $d_{U}+\mathfrak{d}_{\infty}$. Hence, Claim A.3 yields positive real numbers $c_{12}$ and $c_{13}$ such that:

$$
\begin{aligned}
\left|T_{\text {Dist }}\right| \leq c_{12} \Omega\left(z_{r}, x_{r}\right) U(\hat{\mathfrak{X}}, E) & +\frac{c_{\infty} c_{13}}{a_{2}} L \theta(U(\hat{\mathfrak{X}}, E)) \\
& +\sum_{i=1}^{n}\left|\frac{\partial U}{\partial e_{i}}(\hat{\mathfrak{X}}, E)\right| L^{1-i-b} \mu_{i}\left(Z_{i}\right)+\sum_{i=1}^{n}\left|\frac{\partial U}{\partial e_{i}}(\hat{\mathfrak{X}}, E)\right| L^{1-i-b} \delta_{r, i} .
\end{aligned}
$$

Collecting (27), (47) and (48) with $\bar{\Omega} \geq \frac{\Omega}{c_{\infty}}$, we obtain 


$$
\begin{aligned}
\overparen{U(\hat{\mathfrak{X}}, E)} \leq-\left[c_{1}-c_{11} a_{1}-\frac{c_{\infty} c_{13}}{a_{2}}\right] & L \theta(U(\hat{\mathfrak{X}}, E))-\left[c_{2} a_{3}-c_{12} c_{\infty}\right] \bar{\Omega}\left(z_{r}, x_{r}\right) U(\hat{\mathfrak{X}}, E) \\
& +\sum_{i=1}^{n}\left|\frac{\partial U}{\partial e_{i}}(\hat{\mathfrak{X}}, E)\right| L^{1-i-b} \mu_{i}\left(Z_{i}\right)+\sum_{i=1}^{n}\left|\frac{\partial U}{\partial e_{i}}(\hat{\mathfrak{X}}, E)\right| L^{1-i-b} \delta_{r, i} .
\end{aligned}
$$

Hence setting $c_{3}=\frac{c_{1}}{10}$, for all sufficiently small $a_{1}$ and large $a_{2}$ and $a_{3}$, we have

$$
\overparen{U(\hat{\mathfrak{X}}, E)} \leq-5 c_{3} L \theta(U(\hat{\mathfrak{X}}, E))+\sum_{i=1}^{n}\left|\frac{\partial U}{\partial e_{i}}(\hat{\mathfrak{X}}, E)\right| \frac{\mu_{i}\left(Z_{i}\right)}{L^{i+b-1}}+\sum_{i=1}^{n}\left|\frac{\partial U}{\partial e_{i}}(\hat{\mathfrak{X}}, E)\right| \frac{\delta_{r, i}}{L^{i+b-1}}
$$

The function $\left(\hat{\mathfrak{X}}, E, \bar{\delta}_{r, i}\right) \mapsto \frac{\partial U}{\partial e_{i}}(\hat{\mathfrak{X}}, E) \bar{\delta}_{r, i}$ is homogeneous in the bi-limit with degrees $d_{U}$ and $d_{U}+\mathfrak{d}_{\infty}$ and the weights 1 and $r_{\infty, i}+\mathfrak{d}_{\infty}$ for $\bar{\delta}_{r, i}$. Hence there exists a positive real number $c_{4}$ satisfying for any $E, i, Z_{i}$ and $L \geq 1$,

$$
\begin{aligned}
\left|\frac{\partial U}{\partial e_{i}}(\hat{\mathfrak{X}}, E)\right| \frac{\delta_{r, i}}{L^{i+b}} & \leq \frac{c_{3}}{2 n} \theta(U(\hat{\mathfrak{X}}, E))+c_{4} \zeta_{i}\left(\frac{\delta_{r, i}}{L^{i+b}}\right), \\
L^{1-\epsilon}\left|\frac{\partial U}{\partial e_{i}}(\hat{\mathfrak{X}}, E)\right| \frac{\mu_{i}\left(Z_{i}\right)}{L^{i+b-\epsilon}} & \leq L \frac{c_{3}}{2 n} \theta(U(\hat{\mathfrak{X}}, E))+c_{4} L^{1-\epsilon} \zeta_{i}\left(\frac{\mu_{i}\left(Z_{i}\right)}{L^{i+b-\epsilon}}\right),
\end{aligned}
$$

where $\zeta_{i}$ is the homogeneous in the bi-limit functions defined in (31). Hence, inequality (49) becomes (29) and yields the claim of Lemma 1.

\section{B.3 Proof of Lemma [2]}

Before entering the proof of Lemma 2, it is useful to list some properties of the functions $\mu_{i}$ and $\zeta_{i}$. The functions $\mu_{i}^{q_{1}}$ are zero at zero and convex, hence by (7), we have :

$$
\frac{1}{q_{1}} \mu_{i}(s) \leq s \mu_{i}^{\prime}(s) \leq q_{2} \mu_{i}(s) \quad \forall s>0 .
$$

Similarly, by definition (31) of the functions $\zeta_{i}$, we have :

$$
\zeta_{i}(s) \leq s \zeta_{i}^{\prime}(s) \leq \frac{d_{U}+\mathfrak{d}_{\infty}}{1-\mathfrak{d}_{\infty}(n-2)} \zeta_{i}(s) \quad \forall s \geq 0
$$

By integration, this gives :

$$
\zeta_{i}(r s) \leq r^{\frac{d_{U}+\mathrm{d}_{\infty}}{1-\mathrm{d}_{\infty}(n-2)}} \zeta_{i}(s) \quad \forall s \geq 0, \forall r \geq 1
$$

Also, since $d_{U}$ satisfies $\frac{d_{U}-1}{1+\mathfrak{d}_{\infty}}>q_{1}$, the functions $s \mapsto \zeta_{i}\left(\mu_{i}(s)\right)$ are convex, $C^{1}$ and zero at zero. It follows that we have :

$$
\begin{aligned}
& \frac{\partial}{\partial r} \zeta_{i}\left(r \mu_{i}(s)\right)=\zeta_{i}^{\prime}\left(r \mu_{i}(s)\right) \mu_{i}(s), \quad \frac{\partial}{\partial s} \zeta_{i}\left(r \mu_{i}(s)\right)=r \zeta_{i}^{\prime}\left(r \mu_{i}(s)\right) \mu_{i}^{\prime}(s) \text { if } s>0, \\
& =0 \quad \text { if } s=0 \text {, } \\
& 0 \leq \frac{\partial}{\partial s} \zeta_{i}\left(r \mu_{i}\left(s_{1}\right)\right) \leq \frac{\partial}{\partial s} \zeta_{i}\left(r \mu_{i}\left(s_{2}\right)\right) \quad \forall 0 \leq s_{1} \leq s_{2}, \forall r \geq 0
\end{aligned}
$$


Proof of Lemma 2 By (53) and $Y_{i}$ defined in (30), Assumption 1.2 gives :

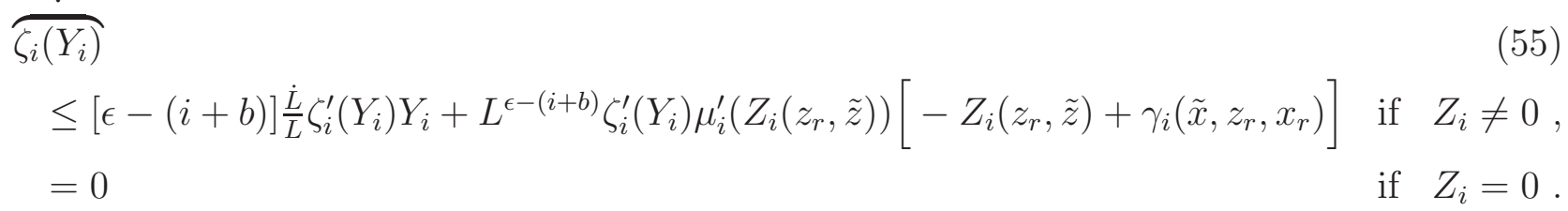

Since $\dot{L}+a_{1} L$ is non-negative and $\epsilon$ satisfies (12), we have :

$$
[\epsilon-(i+b)] \frac{\dot{L}}{L} \zeta_{i}^{\prime}\left(Y_{i}\right) Y_{i} \leq[(i+b)-\epsilon] a_{1} \zeta_{i}^{\prime}\left(Y_{i}\right) Y_{i}
$$

To exhibit an upper bound on $L^{\epsilon-(i+b)} \zeta_{i}^{\prime}\left(Y_{i}\right) \mu_{i}^{\prime}\left(Z_{i}\left(z_{r}, \tilde{z}\right)\right)\left[-Z_{i}\left(z_{r}, \tilde{z}\right)+\gamma_{i}\left(\tilde{x}, z_{r}, x_{r}\right)\right]$ we distinguish two cases :

$$
\text { 1) } \gamma_{i}\left(\tilde{x}, z_{r}, x_{r}\right) \leq \frac{\left.Z_{i}\left(z_{r}, \tilde{z}\right)\right)}{1+v} \quad, \quad \text { 2) } \gamma_{i}\left(\tilde{x}, z_{r}, x_{r}\right)>\frac{\left.Z_{i}\left(z_{r}, \tilde{z}\right)\right)}{1+v}
$$

with $v$ given in Assumption 3.

Case 1) : (50) yields :

$$
\begin{aligned}
L^{\epsilon-(i+b)} \zeta_{i}^{\prime}\left(Y_{i}\right) \mu_{i}^{\prime}\left(Z_{i}\left(z_{r}, \tilde{z}\right)\right)\left[-Z_{i}\left(z_{r}, \tilde{z}\right)+\right. & \left.\gamma_{i}\left(\tilde{x}, z_{r}, x_{r}\right)\right] \\
& \left.\leq-\frac{v}{1+v} L^{\epsilon-(i+b)} \zeta_{i}^{\prime}\left(Y_{i}\right) \mu_{i}^{\prime}\left(Z_{i}\left(z_{r}, \tilde{z}\right)\right) Z_{i}\left(z_{r}, \tilde{z}\right)\right), \\
& \leq-\frac{v}{q_{1}(1+v)} \zeta_{i}^{\prime}\left(Y_{i}\right) L^{\epsilon-(i+b)} \mu_{i}\left(Z_{i}\left(z_{r}, \tilde{z}\right)\right), \\
& \leq-\frac{v}{q_{1}(1+v)} \zeta_{i}^{\prime}\left(Y_{i}\right) Y_{i} .
\end{aligned}
$$

Case 2) : By using successively (54), (50) and (51), we get :

$$
\begin{aligned}
& L^{\epsilon-(i+b)} \zeta_{i}^{\prime}\left(Y_{i}\right) \mu_{i}^{\prime}\left(Z_{i}\left(z_{r}, \tilde{z}\right)\right)\left[-Z_{i}\left(z_{r}, \tilde{z}\right)+\gamma_{i}\left(\tilde{x}, z_{r}, x_{r}\right)\right] \\
& \leq L^{\epsilon-(i+b)} \zeta_{i}^{\prime}\left(L^{\epsilon-(i+b)} \mu_{i}\left((1+v) \gamma_{i}\left(\tilde{x}, z_{r}, x_{r}\right)\right)\right) \mu_{i}^{\prime}\left((1+v) \gamma_{i}\left(\tilde{x}, z_{r}, x_{r}\right)\right) \gamma_{i}\left(\tilde{x}, z_{r}, x_{r}\right)-\frac{1}{q_{1}} \zeta_{i}^{\prime}\left(Y_{i}\right) Y_{i}, \\
& \leq \zeta_{i}^{\prime}\left(L^{\epsilon-(i+b)} \mu_{i}\left((1+v) \gamma_{i}\left(\tilde{x}, z_{r}, x_{r}\right)\right)\right) \frac{q_{2}}{1+v} L^{\epsilon-(i+b)} \mu_{i}\left((1+v) \gamma_{i}\left(\tilde{x}, z_{r}, x_{r}\right)\right)-\frac{1}{q_{1}} \zeta_{i}^{\prime}\left(Y_{i}\right) Y_{i}, \\
& \leq \frac{d_{U}+\mathfrak{d}_{\infty}}{1-\mathfrak{d}_{\infty}(n-2)} \frac{q_{2}}{1+v} \zeta_{i}\left(L^{\epsilon-(i+b)} \mu_{i}\left((1+v) \gamma_{i}\left(\tilde{x}, z_{r}, x_{r}\right)\right)\right)-\frac{1}{q_{1}} \zeta_{i}^{\prime}\left(Y_{i}\right) Y_{i},
\end{aligned}
$$

where by Assumption 3, (26) and $L \geq 1$, we have :

$$
\begin{aligned}
L^{\epsilon-(i+b)} \mu_{i}\left((1+v) \gamma_{i}\left(\tilde{x}, z_{r}, x_{r}\right)\right) & \leq L^{\epsilon-(i+b)} \sum_{j=1}^{i} \Omega\left(z_{r}, x_{r}\right)\left|\tilde{x}_{j}\right|+c_{\infty}\left|\tilde{x}_{j}\right|^{\frac{r_{\infty, i}+\boldsymbol{d} \infty}{r_{\infty, j}}} \\
& \leq \max \left\{\frac{\Omega\left(z_{r}, x_{r}\right)}{c_{\infty}}, 1\right\} c_{\infty} \sum_{j=1}^{i}\left|\hat{\mathcal{X}}_{j}-e_{j}\right|+\left|\hat{\mathcal{X}}_{j}-e_{j}\right|^{\frac{r_{\infty, i}+\boldsymbol{d} \infty}{r_{\infty}, j}} .
\end{aligned}
$$

Since the function $\left(s_{j}\right) \mapsto \sum_{j=1}^{i}\left|s_{j}\right|+\left|s_{j}\right|^{\frac{r_{\infty, i}+\mathrm{d}_{\infty}}{r_{\infty, j}}}$ is homogeneous in the bi-limit with weights $r_{0}$ and $r_{\infty}$ and degrees 1 and $r_{\infty, i}+\mathfrak{d}_{\infty}$, the function $\zeta_{i}$ is homogeneous in the bi-limit with 
weights 1 and $r_{\infty, i}+\mathfrak{d}_{\infty}$ and degrees $d_{U}$ and $d_{U}+\mathfrak{d}_{\infty}$, and the function $(\hat{\mathfrak{X}}, E) \mapsto \theta(U(\hat{\mathfrak{X}}, E))$ is positive definite, proper and homogeneous in the bi-limit with weights $\left(r_{0}, r_{0}\right)$ and $\left(r_{\infty}, r_{\infty}\right)$ and degrees $d_{U}$ and $d_{U}+\mathfrak{d}_{\infty}$, there exists a positive real number $c_{7}$ such that we have :

$$
\frac{d_{U}+\mathfrak{d}_{\infty}}{1-\mathfrak{d}_{\infty}(n-2)} \frac{q_{2}}{1+v} \zeta_{i}\left(c_{\infty} \sum_{j=1}^{i}\left|\hat{\mathcal{X}}_{j}-e_{j}\right|+\left|\hat{\mathcal{X}}_{j}-e_{j}\right|^{\frac{r_{\infty, i}+\mathfrak{d}_{\infty}}{r_{\infty, j}}}\right) \leq c_{7} \theta(U(\hat{\mathfrak{X}}, E))
$$

Hence, with (52), we have obtained :

$$
\begin{aligned}
& \frac{d_{U}+\mathfrak{d}_{\infty}}{1-\mathfrak{d}_{\infty}(n-2)} \frac{q_{2}}{1+v} \zeta_{i}\left(L^{\epsilon-(i+b)} \mu_{i}\left((1+v) \gamma_{i}\left(\tilde{x}, z_{r}, x_{r}\right)\right)\right) \\
& \quad \leq c_{7} \max \left\{\frac{\Omega\left(z_{r}, x_{r}\right)}{c_{\infty}}, 1\right\}^{\frac{d_{U}+\mathfrak{d}_{\infty}}{1-\mathfrak{d}_{\infty}(n-2)}} \theta(U(\hat{\mathfrak{X}}, E)) .
\end{aligned}
$$

Combining the two cases, we get :

$$
\begin{aligned}
L^{\epsilon-(i+b)} \zeta_{i}^{\prime}\left(Y_{i}\right) \mu_{i}^{\prime}\left(Z_{i}\left(z_{r}, \tilde{z}\right)\right)\left[-Z_{i}\left(z_{r}, \tilde{z}\right)+\gamma_{i}\left(\tilde{x}, z_{r}, x_{r}\right)\right] \leq \\
\quad-\frac{v}{q_{1}(1+v)} \zeta_{i}^{\prime}\left(Y_{i}\right) Y_{i}+c_{7} \max \left\{\frac{\Omega\left(z_{r}, x_{r}\right)}{c_{\infty}}, 1\right\}^{\frac{d_{U}+\mathrm{d} \infty}{1-\mathrm{d}_{\infty}(n-2)}} \theta(U(\hat{\mathfrak{X}}, E))
\end{aligned}
$$

which implies for (55) :

$$
\begin{aligned}
\overparen{\zeta_{i}\left(Y_{i}\right)} \leq & -\left(\frac{v}{q_{1}(1+v)}-[(i+b)-\epsilon] a_{1}\right) \zeta_{i}^{\prime}\left(Y_{i}\right) Y_{i} \\
& +c_{7} \max \left\{\frac{\Omega\left(z_{r}, x_{r}\right)}{c_{\infty}}, 1\right\}^{\frac{d_{U}+\mathfrak{d}_{\infty}}{1-\mathfrak{d} \infty(n-2)}} \theta(U(\hat{\mathfrak{X}}, E)), \\
& \leq-c_{6} \zeta_{i}\left(Y_{i}\right)+c_{7} \max \left\{\frac{\Omega\left(z_{r}, x_{r}\right)}{c_{\infty}}, 1\right\}^{\frac{d_{U}+\mathfrak{d}_{\infty}}{1-\mathfrak{d} \infty(n-2)}} \theta(U(\hat{\mathfrak{X}}, E)),
\end{aligned}
$$

where to get the latter inequality we have used (51) and chosen $c_{6}$ satisfying :

$$
c_{6}<\frac{v}{q_{1}(1+v)}
$$

and $a_{1}$ satisfying

$$
a_{1}<\frac{\frac{v}{q_{1}(1+v)}-c_{6}}{n+b} .
$$

This completes the proof of the Lemma. 